TRANSACTIONS OF THE

AMERICAN MATHEMATICAL SOCIETY

Volume 359, Number 7, July 2007, Pages 3505-3528

S 0002-9947(07)04241-9

Article electronically published on February 21, 2007

\title{
MULTIVARIABLE ALEXANDER INVARIANTS OF HYPERSURFACE COMPLEMENTS
}

\author{
ALEXANDRU DIMCA AND LAURENTIU MAXIM
}

\begin{abstract}
We start with a discussion on Alexander invariants, and then prove some general results concerning the divisibility of the Alexander polynomials and the supports of the Alexander modules, via Artin's vanishing theorem for perverse sheaves. We conclude with explicit computations of twisted cohomology following an idea already exploited in the hyperplane arrangement case, which combines the degeneration of the Hodge to de Rham spectral sequence with the purity of some cohomology groups.
\end{abstract}

\section{INTRODUCTION}

Alexander invariants in the form of Alexander modules, characteristic varieties and Alexander polynomials have been recently intensively studied, in particular in relation to the twisted cohomology of hypersurface arrangement complements; see for instance [1, 44, [5], 6], [18, [20], 21], 22], 25], 32], [33, 36], 42], 47].

In section 2, after giving the basic definitions introducing the Alexander modules $A^{q}(\mathcal{U})$ and $A_{q}(\mathcal{U})$ of an affine hypersurface arrangement complement $\mathcal{U}$, we investigate in Proposition 2.4 the relation between the first nontrivial Alexander polynomial in one variable and the corresponding Alexander polynomial in several variables. Proposition 2.5 expresses the relation between the characteristic varieties defined using the Fitting ideals and the characteristic varieties defined using the jumping loci of the cohomology with rank one local coefficients. Example 2.8 treats the simplest local situations: the normal crossing case and the case of isolated non-normal crossing singularities, whose study was initiated by A. Libgober in [36].

In section 3, Theorem 3.1 relates the Alexander invariants of the affine hypersurface arrangement complement $\mathcal{U}=\mathbb{C}^{n+1} \backslash X$ to the Alexander invariants of the complement $\mathcal{U}_{\infty}$ of the corresponding link at infinity. Theorems 3.2, 3.6 and Corollary 3.5 estimate the support of the Alexander modules $A^{q}(\mathcal{U})$ in terms of local properties of the projective closure $V=\bar{X}$.

In section 4, we recall and slightly extend the idea of combining the degeneration of the Hodge to de Rham spectral sequence with the purity of some cohomology groups (used first by Esnault, Schechtman and Viehweg in 25] and by Schechtman, Terao and Varchenko in 47); see Corollary 4.1 and Proposition 4.5. Examples 4.8 and 4.10 illustrate this approach by looking at some arrangements of lines and conics in the plane. Though these examples may be treated using the results by

Received by the editors August 25, 2005.

2000 Mathematics Subject Classification. Primary 32S20, 32S22, 32S35, 32S60; Secondary 14J70, 14F17, 14F 45.

(C)2007 American Mathematical Society Reverts to public domain 28 years from publication 3505 
Cogolludo in 4, we feel that our approach is more general and hence more likely to extend to other situations.

In the last section we consider the complement $\mathcal{U}_{0}$ of an arbitrary projective hypersurface arrangement $V$, and, after a short general discussion, we revisit from a new perspective a useful result by Randell saying what happens to the twisted cohomology of a plane curve complement when we add an extra line; see Corollary 5.1. Coming back to dimension $n \geq 2$, Example 5.3 discusses the already interesting case when $V$ is irreducible and has only isolated singularities. This case leads, in particular, to examples where for $m=n, n+1$ and some rank one local coefficients $\mathcal{L}_{\beta}$ on $\mathcal{U}_{0}$ one has

$$
\operatorname{dim} H^{m}\left(\mathcal{U}_{0}, \mathcal{L}_{\beta}\right)>\operatorname{dim} H^{m}\left(\mathcal{U}_{0}, \mathbb{C}\right) .
$$

By the minimality property of hyperplane arrangement complements, it is known that the above inequality is impossible for such type of complements, 23. We conclude by a detailed study of the case when $V$ has two irreducible components, each of them having only isolated singularities.

Throughout the paper we usually work with complex coefficients $\mathbb{C}$, although the study of finite field coefficients is very important, due for instance to torsion open questions; see [8, 41]. Our choice is imposed by the analytic tools used in the last two sections. Most of the results in the previous sections hold over arbitrary fields.

\section{Multivariable Alexander invariants}

2.1. Algebraic preliminaries. Let $R$ be a commutative ring with unit, which is Noetherian and a unique factorization domain (e.g., the ring of complex Laurent polynomials in $s$ variables, $s \geq 1$ ). Let $A$ be a finitely generated $R$-module, and $M$ an $(n \times m)$ presentation matrix of $A$ associated to an exact sequence

$$
R^{m} \rightarrow R^{n} \rightarrow A \rightarrow 0 .
$$

The $i$-th elementary ideal $\mathcal{E}_{i}(A)$ of $A$ is the ideal in $R$ generated by the $(n-i) \times(n-i)$ minor determinants of $M$, with the convention that $\mathcal{E}_{i}(A)=R$ if $i \geq n$, and $\mathcal{E}_{i}(A)=0$ if $n-i>m$. Let $\Delta_{i}(A)$ be the generator of the smallest principal ideal in $R$ containing $\mathcal{E}_{i}(A)$, i.e., the greatest common divisor of all elements of $\mathcal{E}_{i}(A)$. $\Delta_{i}(A)$ is called the $i$-th characteristic polynomial of $A$. Note that $\Delta_{i+1}(A)$ divides $\Delta_{i}(A)$ in $R$ for all $i$ since $\mathcal{E}_{i}(A) \subset \mathcal{E}_{i+1}(A)$. In particular, if $R$ is a principal ideal domain (e.g., the ring of complex Laurent polynomials in one variable), then $\mathcal{E}_{i}(A)$ is a principal ideal generated exactly by $\Delta_{i}(A)$.

As an example, for any ring $R$, assume that $A=R^{s} \oplus R /\left(\lambda_{1}\right) \oplus \cdots \oplus R /\left(\lambda_{r}\right)$, where $\lambda_{j}(j=1,2, \cdots, r)$ are nonzero elements in $R$ such that $\lambda_{j+1} \mid \lambda_{j}$. Then we have $\Delta_{i}(A)$ is $0, \lambda_{i-s+1} \cdots \lambda_{r}$, or 1 , according to whether $0 \leq i \leq s-1, s \leq i \leq s+r-1$, or $s+r \leq i$.

The support $\operatorname{Supp}(A)$ of $A$ is the reduced subscheme of $\operatorname{Spec}(R)$ defined by (the order ideal) $\mathcal{E}_{0}(A)$. Since

$$
\sqrt{\mathcal{E}_{0}(A)}=\sqrt{\operatorname{Ann}(A)}
$$

this is the usual notion of support in algebraic geometry based on the annihilator ideal $\operatorname{Ann}(A)$ of the module $A$. In particular, for a prime ideal $P \subset R, P \in \operatorname{Supp}(A)$ if and only if the localized module $A_{P}$ is nonzero.

The support $\operatorname{Supp}(A)$ is also called the first characteristic variety of $A$, and we define the $i$-th characteristic variety $V_{i}(A)$ of $A$ to be the reduced subscheme of $\operatorname{Spec}(R)$ defined by the $\left(i\right.$-th Fitting ideal) ideal $\mathcal{E}_{i-1}(A)$. 
Note that $\operatorname{codim} V_{i}(A)>1$ implies $\Delta_{i-1}(A)=1$; i.e., the corresponding Alexander polynomial carries no information.

All definitions above are independent (up to multiplication by a unit of $R$ ) of the choices involved; thus the characteristic varieties and polynomials of $A$ are invariants of the $R$-isomorphism type of $A$.

We state for future reference the following "divisibility" properties of the polynomials and characteristic varieties (for proofs, see [50 and [35]):

Lemma 2.1. $\quad$ - If $A, B$ are finitely generated $R$-modules, then

$$
\Delta_{0}(A \oplus B)=\Delta_{0}(A) \times \Delta_{0}(B) .
$$

- If $A$ and $B$ are finitely generated $R$-modules, then

$$
\operatorname{Supp}\left(A \otimes_{R} B\right)=\operatorname{Supp}(A) \cap \operatorname{Supp}(B) .
$$

- If $A$ is a submodule of $B$, then for all $i, \Delta_{i}(A)$ divides $\Delta_{i}(B)$.

- If $0 \rightarrow A \rightarrow B \rightarrow C \rightarrow 0$ is a short exact sequence of finitely generated $R$-modules, then the following hold:

(1) $\Delta_{0}(B)=\Delta_{0}(A) \times \Delta_{0}(C)$;

(2) for all $i, \Delta_{i}(B)$ divides $\Delta_{i}(A) \times \Delta_{0}(C)$;

(3) if $\Delta_{0}(C)=1$, then $\Delta_{i}(A)=\Delta_{i}(B)$ for all $i$;

(4) $\operatorname{Supp}(B)=\operatorname{Supp}(A) \cup \operatorname{Supp}(C)$;

(5) for $i \geq 2$ : $V_{i}(C) \subset V_{i}(B) \subset V_{i}(C) \cup\left(V_{i-1}(C) \cap \operatorname{Supp}(A)\right)$.

2.2. Alexander invariants of hypersurface complements. Let $V$ be a reduced hypersurface in $\mathbb{C P}^{n+1}$, defined by a homogeneous equation: $f=f_{1} \cdots f_{s}=0$, where the $f_{i}$ are the irreducible factors of $f$, and $V_{i}=\left\{f_{i}=0\right\}$ the irreducible components of $V$. We fix a hyperplane $H$ in $\mathbb{C P}^{n+1}$, which we call "the hyperplane at infinity". Let $\mathcal{U}$ be the (affine) hypersurface complement $\mathcal{U}=\mathbb{C P}^{n+1} \backslash(V \cup H)$. (Alternatively, $\mathcal{U}$ may be regarded as the complement of a hypersurface in the affine space $\mathbb{C}^{n+1}$.) Then $H_{1}(\mathcal{U}) \cong \mathbb{Z}^{s}$ ([16, (4.1.3), (4.1.4)), generated by the meridian loops $\gamma_{i}$ about the nonsingular part of each irreducible component $V_{i}$, for $i=1, \cdots, s$. If $\gamma_{\infty}$ denotes the meridian about the hyperplane at infinity, then in $H_{1}(\mathcal{U})$ there is a relation: $\gamma_{\infty}+\sum d_{i} \gamma_{i}=0$, where $d_{i}=\operatorname{deg}\left(V_{i}\right)$.

Note that $\mathcal{U}$ is affine, therefore has the homotopy type of a finite $\mathrm{CW}$ complex. Let $\mathcal{U}^{a b}$ be the universal abelian cover of $\mathcal{U}$, i.e. the covering associated to the commutator subgroup of $\pi_{1}(\mathcal{U})$, or equivalently, the covering associated to the kernel of the linking number homomorphism $l k: \pi_{1}(\mathcal{U}) \rightarrow \mathbb{Z}^{s}$, which maps a loop $\alpha$ to $\left(\operatorname{lk}\left(\alpha, V_{1} \cup-d_{1} H\right), \cdots, \operatorname{lk}\left(\alpha, V_{s} \cup-d_{s} H\right)\right)$. The group of covering transformations of $\mathcal{U}^{a b}$ is isomorphic to $\mathbb{Z}^{s}$ and acts on the covering space. By choosing fixed lifts of the cells of $\mathcal{U}$ to $\mathcal{U}^{a b}$, we obtain a free basis for $C_{*}$, the cellular cell complex of $\mathcal{U}^{a b}$, as a $\mathbb{Z}\left[\mathbb{Z}^{s}\right]$-module. The isomorphism determined by the meridians $\left\{\gamma_{i}\right\}$ enables us to identify $\mathbb{Z}\left[\mathbb{Z}^{s}\right]$ with $\mathbb{Z}\left[t_{1}, t_{1}^{-1}, \cdots, t_{s}, t_{s}^{-1}\right]$, the ring of integral Laurent polynomials in $s$ variables. When $s=1$ we set $t_{1}=t$.

For reasons that will become transparent later, our base ring will always be the ring of complex Laurent polynomials in $s$ variables, $\mathbb{C}\left[t_{1}, t_{1}^{-1}, \cdots, t_{s}, t_{s}^{-1}\right]$, which we denote by $R_{s}$. Note that $R_{s}$ is a regular Noetherian domain, and in particular it is factorial. As a group ring, $R_{s}$ has a natural involution denoted by an overbar, sending each $t_{i}$ to $\bar{t}_{i}:=t_{i}^{-1}$. To an $R_{s}$-module $A$, we associate the conjugate $R_{s^{-}}$ module, still denoted by $A$, with the same underlying abelian group but with the $R_{s}$-action given by $(r, a) \mapsto \bar{r} \cdot a$, for $a \in A$ and $r \in R_{s}$. 
Remark 2.2. Though the ring $R_{s}$ is commutative, it should be regarded as a quotient ring of $\mathbb{C}\left[\pi_{1}(\mathcal{U})\right]$, which is non-commutative in general. Because of that, one should be careful to distinguish the right from the left $R_{s}$-modules. If, for instance, $A$ is a left $R_{s}$-module, then the associated right $R_{s}$-module is the module conjugate to $A$, whose module structure is given by

$$
a \cdot r:=\bar{r} \cdot a
$$

for all $a \in A$ and $r \in R_{s}$. This corresponds to regarding any left $\mathbb{C}\left[\pi_{1}(\mathcal{U})\right]$-module $A$ as a right $\mathbb{C}\left[\pi_{1}(\mathcal{U})\right]$-module by setting $a \cdot \gamma=\gamma^{-1} \cdot a$, for all $a \in A$ and $\gamma \in \pi_{1}(\mathcal{U})$, and extending by linearity. Following [11, p. 97, we regard in this paper $C_{*}^{0}=C_{*} \otimes \mathbb{C}$ as a complex of right $R_{s}$-modules.

Define a local coefficient system $\mathcal{L}$ on $\mathcal{U}$, with stalk $R_{s}$ and action of a loop $\alpha \in$ $\pi_{1}(\mathcal{U})$ determined by (left) multiplication by $\prod_{j=1}^{s}\left(t_{j}\right)^{1 \mathrm{k}\left(\alpha, V_{j} \cup-d_{j} H\right)}$. In particular, the action of the meridian $\gamma_{i}$ is given by multiplication by $t_{i}$. Let $\mathcal{L}^{\vee}$ be the dual local system, whose stalk at a point $y \in \mathcal{U}$ is $\mathcal{L}_{y}^{\vee}:=\operatorname{Hom}\left(\mathcal{L}_{y}, R_{s}\right)$, and let $\alpha \in \pi_{1}(\mathcal{U}, y)$ act on $\varphi \in \mathcal{L}_{y}^{\vee}$ by

$$
(\alpha \cdot \varphi)(m):=\varphi\left(\alpha^{-1} \cdot m\right), \quad m \in \mathcal{L}_{y} .
$$

We denote by $\overline{\mathcal{L}}$ the local system obtained from $\mathcal{L}$ by composing all module structures with the involution of $R_{s}$ (i.e., by changing the stalks of $\mathcal{L}$ from left into right $R_{s}$-modules). The perfect pairing

$$
\overline{\mathcal{L}} \otimes_{R_{s}} \mathcal{L} \rightarrow R_{s}
$$

given by

$$
(f, g) \mapsto \bar{f} \cdot g
$$

on the stalk over a basepoint, tells us that there is an isomorphism of local systems on $\mathcal{U}$ :

$$
\mathcal{L}^{\vee} \simeq \overline{\mathcal{L}}
$$

The universal homology $k$-th Alexander invariant $A_{k}(\mathcal{U})$ of $\mathcal{U}$ is by definition the $R_{s}$-module $H_{k}\left(C_{*}^{0}\right)$, or equivalently $H_{k}(\mathcal{U} ; \mathcal{L})$. This is the group $H_{k}\left(\mathcal{U}^{a b} ; \mathbb{C}\right)$ considered as an $R_{s}$-module via the covering transformations (see 29], Example 3 H.2). Similarly, the universal cohomology $k$-th Alexander invariant $A^{k}(\mathcal{U})$ of $\mathcal{U}$ is by definition the $k$-th cohomology module of the dual complex $\operatorname{Hom}_{R_{s}}\left(C_{*}^{0}, R_{s}\right)$. Here $R_{s}$ is considered with the induced right $R_{s}$-module structure as explained in Remark 2.2. Based on our previous considerations on local systems, $A^{k}(\mathcal{U})$ is just $H^{k}\left(\mathcal{U} ; \mathcal{L}^{\vee}\right)$. This may also be regarded as the $k$-th cohomology with compact support and complex coefficients of $\mathcal{U}_{b}^{a b}$, where $\mathcal{U}_{b}$ is the compact manifold with boundary obtained from $\mathbb{C P}^{n+1}$ by removing a small open regular neighborhood of the divisor $V \cup H$ (compare [29], Prop. 3H.5).

Note that, since $\mathcal{U}$ is an $(n+1)$-dimensional affine variety, the modules $A^{k}(\mathcal{U})$ and resp. $A_{k}(\mathcal{U})$ are trivial for $k>n+1$. Moreover, since the stalks of $\mathcal{L}$ are torsion-free, $A_{n+1}(\mathcal{U})$ is also a torsion-free $R_{s}$-module (see [48, Example 6.0.6).

As in classical knot theory, by using a deformation retract argument, one could define the universal abelian invariants above after replacing $\mathcal{U}$ by the manifold with boundary $\mathcal{U}_{b}$, obtained from $\mathbb{C P}^{n+1}$ by removing a small open regular neighborhood of the divisor $V \cup H$. Now, since the chain complex $C_{*}\left(\mathcal{U}_{b}^{a b}\right)$ is of finite type, and since $R_{s}$ is Noetherian, this implies that all these universal Alexander modules are finitely generated. Hence their characteristic varieties and polynomials are well 
defined. The associated characteristic varieties, in particular the supports, become subvarieties of the $s$-dimensional torus $\mathbb{T}^{s}=\left(\mathbb{C}^{*}\right)^{s}$, which is regarded as the set of closed points in $\operatorname{Spec}\left(R_{s}\right)$. More precisely, for $\lambda=\left(\lambda_{1}, \cdots, \lambda_{s}\right) \in \mathbb{T}^{s}$, we denote by $m_{\lambda}$ the corresponding maximal ideal in $R_{s}$ and by $\mathbb{C}_{\lambda}$ the quotient $R_{s} / m_{\lambda} R_{s}$. This quotient is isomorphic to $\mathbb{C}$, and the canonical projection

$$
\rho_{\lambda}: R_{s} \rightarrow R_{s} / m_{\lambda} R_{s}=\mathbb{C}_{\lambda}
$$

corresponds to replacing $t_{j}$ by $\lambda_{j}$ for $j=1, \ldots, s$. Here we regard $\mathbb{C}_{\lambda}$ as a (left) $R_{s}$-module, with an involution given by complex conjugation (which is compatible with the one induced from $R_{s}$ since $\lambda_{j} \in \mathbb{T}^{1}$ ).

If $A$ is an $R_{s}$-module, we denote be $A_{\lambda}$ the localization of $A$ at the maximal ideal $m_{\lambda}$. For $A=R_{s}$, we use the simpler notation $R_{\lambda}$ when there is no danger of confusion. If $A$ is of finite type, then $A=0$ if and only if $A_{\lambda}=0$ for all $\lambda \in \mathbb{T}^{s}$. More precisely

$$
\operatorname{Supp}(A)=\left\{\lambda \in \mathbb{T}^{s} ; A_{\lambda} \neq 0\right\} .
$$

In particular $A_{0}(\mathcal{U})=\mathbb{C}_{\mathbf{1}}$, where $\mathbf{1}=(1, \cdots, 1)$ and hence

$$
\operatorname{Supp}\left(A_{0}(\mathcal{U})\right)=\{\mathbf{1}\} .
$$

We denote by $V_{i, k}(\mathcal{U})$ the $i$-th characteristic variety associated with the homological Alexander module $A_{k}(\mathcal{U})$, and similarly denote by $\Delta_{i, k}(\mathcal{U})$ the associated characteristic polynomials. The notation $V^{i, k}(\mathcal{U})$ and $\Delta^{i, k}(\mathcal{U})$ denote the similar objects associated with the cohomological Alexander invariants $A^{k}(\mathcal{U})$.

2.3. Homology versus cohomology Alexander modules. It is natural to ask what are the relations between the homology and the cohomology universal Alexander modules, or to find the relations between $V_{i, k}(\mathcal{U})$ and $V^{i, k}(\mathcal{U})$; and between $\Delta_{i, k}(\mathcal{U})$ and $\Delta^{i, k}(\mathcal{U})$.

Some answers to this question can be given as follows. The cohomology modules may be related to the homology modules by the Universal Coefficient spectral sequence (see [30], p.20 or 31], Thm. 2.3):

$$
\operatorname{Ext}_{R_{s}}^{q}\left(A_{p}(\mathcal{U}), R_{s}\right) \Rightarrow A^{p+q}(\mathcal{U}) .
$$

Using the exactness of the localization (see [51], p. 76), we get the following spectral sequence for any $\lambda \in \mathbb{T}^{s}$ :

$$
\operatorname{Ext}_{R_{\lambda}}^{q}\left(A_{p}(\mathcal{U})_{\lambda}, R_{\lambda}\right) \Rightarrow A^{p+q}(\mathcal{U})_{\lambda} .
$$

For a fixed $\lambda \in \mathbb{T}^{s}$, we define

$$
k(\lambda)=\min \left\{m \in \mathbb{N} ; A_{m}(\mathcal{U})_{\lambda} \neq 0\right\} .
$$

Then the spectral sequence (2.4) implies the following.

Proposition 2.3. For any $\lambda \in \mathbb{T}^{s}, A^{k}(\mathcal{U})_{\lambda}=0$ for $k<k(\lambda)$ and

$$
A^{k(\lambda)}(\mathcal{U})_{\lambda}=\operatorname{Hom}\left(A_{k(\lambda)}(\mathcal{U})_{\lambda}, R_{\lambda}\right) .
$$

This equality shows in particular that one may have $A^{k(\lambda)}(\mathcal{U})_{\lambda}=0$, even when $A_{k(\lambda)}(\mathcal{U})_{\lambda} \neq 0$, e.g. when the last module is torsion, which is often the case, e.g. see (2.2). 
2.4. Multivariable versus one variable Alexander modules. Consider a family of integral weights $\mathbf{e}=\left(e_{1}, \cdots, e_{s}\right) \in \mathbb{Z}^{s}$, and let

$$
q:=\text { g.c.d. }\left(e_{1}, \cdots, e_{s}\right) \text {. }
$$

Consider the morphism $p(\mathbf{e}): R_{s} \rightarrow R_{1}$ defined by $t_{i} \mapsto t^{e_{i}}$, inducing a (left) $R_{s^{-}}$ module structure on $R_{1}$. Let $\mathcal{L}(\mathbf{e})$ be the local system on $\mathcal{U}$ with stalk $R_{1}$ and monodromy action for a loop $\alpha \in \pi_{1}(\mathcal{U})$ given by multiplication by $t^{\sum e_{j} \operatorname{lk}\left(\alpha, V_{j} \cup-d_{j} H\right)}$.

The corresponding homology groups $H_{k}(\mathcal{U}, \mathcal{L}(\mathbf{e}))=H_{k}\left(C_{*}^{0} \otimes_{R_{s}} R_{1}\right)$ are finite type $R_{1}$-modules, and hence they have associated characteristic varieties $V_{i, k}(\mathcal{U}, \mathbf{e})$ and Alexander polynomials $\Delta_{i, k}(\mathcal{U}, \mathbf{e})$.

It is natural to ask under which conditions the equalities

$$
\Delta_{i, k}(\mathcal{U}, \mathbf{e})(t)=\left(t^{q}-1\right) \Delta_{i, k}(\mathcal{U})\left(t^{e_{1}}, \cdots, t^{e_{s}}\right)
$$

do hold? Something like this works in classical knot theory, more precisely for oriented multilinks in $S^{3}$ with at least 2 components, where the case $i=0, k=1$ is considered (see [24, Prop. 5.1, and also [43], Lemma 10.1 for the case of weight $(1, \cdots, 1))$.

For the weight $\mathbf{1}=(1,1, \ldots, 1)$, we call the corresponding Alexander polynomials the usual (or, univariable) Alexander polynomials and we denote them by $\Delta_{i, k}^{T}(\mathcal{U})$ (see below for some explanation).

If the equality in Question 2 holds for all but finitely many multi-indices e, then the 1-variable polynomials $\Delta_{i, k}(\mathcal{U}, \mathbf{e})$ determine (up to a unit in $R_{s}$ ) the multivariable polynomial $\Delta_{i, k}(\mathcal{U})$ (see [3] Lemma 2.2).

Some insight into this question can be obtained as follows. We consider only the simplest case, namely $\mathbf{e}=\mathbf{1}$, and leave the other cases to the interested reader.

Note that the universal abelian covering $\mathcal{U}^{a b} \rightarrow \mathcal{U}$ corresponds to the kernel $K^{a b}$ of the abelianization morphism

$$
\pi_{1}(\mathcal{U}) \rightarrow H_{1}(\mathcal{U})
$$

The total linking number covering $\mathcal{U}^{T} \rightarrow \mathcal{U}$ corresponds to the kernel $K^{T}$ of the morphism

$$
\pi_{1}(\mathcal{U}) \rightarrow H_{1}(\mathcal{U})=\mathbb{Z}^{s} \rightarrow \mathbb{Z}
$$

where the second morphism is $\sum c_{j} \gamma_{j} \mapsto \sum c_{j}$. It follows that $\mathcal{U}^{a b} \rightarrow \mathcal{U}^{T}$ is a covering with deck transformation group $G=K^{T} / K^{a b}$ identified to the subgroup

$$
\left\{c \in \mathbb{Z}^{s} ; \sum c_{j}=0\right\} \text {. }
$$

The complex $C_{*}^{0}$ is a complex of free $R_{s}$-modules of finite rank, and the derivatives are $R_{s}$-linear. It follows that we can regard this complex as being a complex $\mathcal{C}_{*}^{0}$ of free $\mathcal{O}_{\mathbb{T}^{s}}$-modules on the affine variety $\mathbb{T}^{s}$.

Since $\mathcal{U}^{T}=\mathcal{U}^{a b} / G$, it follows that the complex of singular chains of $\mathcal{U}^{T}$ is

$$
C_{*}\left(\mathcal{U}^{T}\right)=C_{*}\left(\mathcal{U}^{a b}\right)_{G}=\left(C_{*}^{0}\right)_{G}
$$

(see [51, p.204). Here

$$
\left(C_{p}^{0}\right)_{G}=C_{p}^{0} /\left\langle g m-m ; g \in G, m \in C_{p}^{0}\right\rangle .
$$

Using the fact that the group $G$ is generated by the elements having a 1 as the $i$-th coordinate, a -1 as the $j$-th coordinate (for $i<j$ ) and all the other coordinates zero, we see that $\left(C_{p}^{0}\right)_{G}$ is the quotient of $C_{p}^{0}$ by the submodule

$$
\left\langle\left(t_{i}-t_{j}\right) m ; m \in C_{p}^{0}\right\rangle .
$$


It follows that the associated sheaf $\left(\mathcal{C}_{p}^{0}\right)_{G}$ is just the restriction (as a coherent sheaf) of $\mathcal{C}_{p}^{0}$ to the 1 -dimensional subtorus $S=\left\{(t, t, \ldots, t) \in \mathbb{T}^{s}\right\}$, i.e. $\left(\mathcal{C}_{p}^{0}\right)_{G}=\mathcal{C}_{p}^{0} \otimes_{\mathcal{O}_{\mathbb{T}^{s}}} \mathcal{O}_{S}$. Unfortunately, the inclusion $S \rightarrow \mathbb{T}^{s}$ is not a flat morphism (see [28], p. 254), and hence the restriction to $S$ does not commute with taking homology.

However, by our discussion above,

$$
\left(C_{p}^{0}\right)_{G}=C_{p}^{0} \otimes_{R_{s}} R_{1},
$$

with the (left) $R_{s}$-module structure on $R_{1}$ induced by $p(\mathbf{1})$. Use now the Künneth spectral sequence (see [51, p.143), and get

$$
E_{p, q}^{2}=\operatorname{Tor}_{p}^{R_{s}}\left(A_{q}(\mathcal{U}), R_{1}\right) \Rightarrow H_{p+q}\left(\left(C_{*}^{0}\right)_{G}\right)=A_{p+q}^{T}(\mathcal{U}) .
$$

For $a \in \mathbb{T}^{1}=S=\left\{(t, t, \ldots, t) \in \mathbb{T}^{s}\right\}$, we get by localization a new Künneth spectral sequence, namely,

$$
E_{p, q}^{2}=\operatorname{Tor}_{p}^{R_{a}}\left(A_{q}(\mathcal{U})_{a}, R_{1, a}\right) \Rightarrow H_{p+q}\left(\left(C_{*}^{0}\right)_{G}\right)_{a} .
$$

In particular we get the following.

Proposition 2.4. For any $a \in \mathbb{T}^{1}, A_{k}^{T}(\mathcal{U})_{a}=0$ for $k<k(a)$ and

$$
A_{k(a)}(\mathcal{U})_{a} \otimes_{R_{a}} R_{1, a}=A_{k(a)}^{T}(\mathcal{U})_{a} .
$$

In particular, for any $a \in \mathbb{T}^{1}=S$, the multiplicity of the root $t=a$ in the polynomials $\Delta_{i, k(a)}^{T}(\mathcal{U})(t)$ and $\Delta_{i, k(a)}(\mathcal{U})(t, \cdots, t)$ is the same.

Proof. To get the second claim, note that any presentation

$$
R_{a}^{m} \rightarrow R_{a}^{n} \rightarrow A_{k(a)}(\mathcal{U})_{a} \rightarrow 0
$$

yields by tensor product a presentation

$$
R_{1, a}^{m} \rightarrow R_{1, a}^{n} \rightarrow A_{k(a)}^{T}(\mathcal{U})_{a} \rightarrow 0
$$

2.5. Characteristic varieties as jumping loci of rank-1 local systems. Let $\lambda=\left(\lambda_{1}, \cdots, \lambda_{s}\right) \in \mathbb{T}^{s}$ and denote by $\mathcal{L}_{\lambda}$ the local coefficient system on $\mathcal{U}$ with stalk $\mathbb{C}=\mathbb{C}_{\lambda}$ and action of a loop $\alpha \in \pi_{1}(\mathcal{U})$ determined by multiplication by $\prod_{j=1}^{s}\left(\lambda_{j}\right)^{\mathrm{lk}\left(\alpha, V_{j} \cup-d_{j} H\right)}$. We let $\mathcal{L}_{\lambda}^{\vee} \simeq \mathcal{L}_{\lambda^{-1}}$ be the dual local system, where $\lambda^{-1}:=$ $\left(\lambda_{1}^{-1}, \cdots, \lambda_{s}^{-1}\right) \in \mathbb{T}^{s}$.

One can define new topological characteristic varieties by setting

$$
V_{i, k}^{t}(\mathcal{U})=\left\{\lambda \in \mathbb{T}^{s} ; \operatorname{dim} H_{k}\left(\mathcal{U}, \mathcal{L}_{\lambda}\right)>i\right\}
$$

and

$$
V_{t}^{i, k}(\mathcal{U})=\left\{\lambda \in \mathbb{T}^{s} ; \operatorname{dim} H^{k}\left(\mathcal{U}, \mathcal{L}_{\lambda}\right)>i\right\}
$$

It is natural to investigate the relations between the two types of characteristic varieties. Some cases are considered in 35, 36.

Here is a general approach to this question. It is known that

$$
H_{k}\left(\mathcal{U}, \mathcal{L}_{\lambda}\right)=H_{k}\left(C_{*}^{0} \otimes_{R_{s}} \mathbb{C}_{\lambda}\right)
$$

Using the Künneth spectral sequence, we get

$$
E_{p, q}^{2}=\operatorname{Tor}_{p}^{R_{s}}\left(A_{q}(\mathcal{U}), \mathbb{C}_{\lambda}\right) \Rightarrow H_{p+q}\left(\mathcal{U}, \mathcal{L}_{\lambda}\right)
$$


Now since the localization is exact, the base change for Tor under $R_{s} \rightarrow R_{\lambda}$ (see [51, p. 144), yields a new spectral sequence

$$
E_{p, q}^{2}=\operatorname{Tor}_{p}^{R_{\lambda}}\left(A_{q}(\mathcal{U})_{\lambda}, \mathbb{C}_{\lambda}\right) \Rightarrow H_{p+q}\left(\mathcal{U}, \mathcal{L}_{\lambda}\right) .
$$

This proves the first claim of the next result.

Proposition 2.5. For any point $\lambda \in \mathbb{T}^{s}$, one has the following:

(i) $\min \left\{m \in \mathbb{N}, H_{m}\left(\mathcal{U}, \mathcal{L}_{\lambda}\right) \neq 0\right\}=\min \left\{m \in \mathbb{N}, \lambda \in \operatorname{Supp}\left(A_{m}(\mathcal{U})\right)\right\}=k(\lambda)$;

(ii) $\operatorname{dim} H_{k(\lambda)}\left(\mathcal{U}, \mathcal{L}_{\lambda}\right)=\max \left\{m \in \mathbb{N}, \lambda \in V_{m, k(\lambda)}(\mathcal{U})\right\}$.

Proof. To prove the second claim, note that the spectral sequence (2.13) yields

$$
H_{k(\lambda)}\left(\mathcal{U}, \mathcal{L}_{\lambda}\right)=A_{k(\lambda)}(\mathcal{U})_{\lambda} / m_{\lambda} A_{k(\lambda)}(\mathcal{U})_{\lambda} .
$$

Let $n$ be the dimension of these two vector spaces. Then by Nakayama's Lemma, the module $A_{k(\lambda)}(\mathcal{U})_{\lambda}$ is generated by $n$ elements over the local ring $R_{\lambda}$. In other words, there is the presentation

$$
R_{\lambda}^{m} \rightarrow R_{\lambda}^{n} \rightarrow A_{k(\lambda)}(\mathcal{U})_{\lambda} \rightarrow 0 .
$$

Moreover, the first morphism is given by a matrix $M$ whose entries $m_{i j}$ are all in the maximal ideal $m_{\lambda}$. The second claim now follows by the definition of the characteristic varieties.

Remark 2.6. Note that there is also a spectral sequence

$$
E_{2}^{p, q}=\operatorname{Ext}_{R_{\lambda}}^{q}\left(A_{p}(\mathcal{U})_{\lambda}, \mathbb{C}_{\lambda}\right) \Rightarrow H^{p+q}\left(\mathcal{U}, \mathcal{L}_{\lambda^{-1}}\right) .
$$

Here $\mathbb{C}_{\lambda}$ is considered with the right $R_{s}$-module structure as indicated in Remark 2.2. This is why in the abutment of the spectral sequence (2.14), we obtain a cohomology with coefficients in the dual local system $\mathcal{L}_{\lambda}^{\vee} \simeq \mathcal{L}_{\lambda^{-1}}$. The above spectral sequence yields that $H^{m}\left(\mathcal{U}, \mathcal{L}_{\lambda^{-1}}\right)=0$ for $m<k(\lambda)$ and $H^{k(\lambda)}\left(\mathcal{U}, \mathcal{L}_{\lambda^{-1}}\right)=$ $\operatorname{Hom}_{R_{\lambda}}\left(A_{k(\lambda)}(\mathcal{U})_{\lambda}, \mathbb{C}_{\lambda}\right)$. However

$$
\operatorname{Hom}_{R_{\lambda}}\left(A_{k(\lambda)}(\mathcal{U})_{\lambda}, \mathbb{C}_{\lambda}\right)=\operatorname{Hom}_{\mathbb{C}}\left(A_{k(\lambda)}(\mathcal{U})_{\lambda} / m_{\lambda} A_{k(\lambda)}(\mathcal{U})_{\lambda}, \mathbb{C}_{\lambda}\right)
$$

and hence

$$
H_{k(\lambda)}\left(\mathcal{U}, \mathcal{L}_{\lambda}\right)^{*}=H^{k(\lambda)}\left(\mathcal{U}, \mathcal{L}_{\lambda^{-1}}\right)
$$

(compare [18, p.50 and p. 69). The case $k=1$ of this useful formula was established in [41, Remark 5.2. Note that this formula holds over arbitrary fields, with the same proof as above.

Remark 2.7. All the results in this section so far hold for the local setting as well, i.e., when $\mathcal{U}$ is the complement of a hypersurface germ in a small ball. The first part of the example below corresponds to the germ of a normal crossing divisor. The second part of the example below corresponds to isolated non-normal crossing divisors (for short INNC); see [22], 36], 37].

Similarly, instead of localizing at a point, one may localize along the hyperplane $H$ at infinity, i.e. replace $\mathcal{U}$ by $\mathcal{U}_{\infty}=\mathcal{U} \cap S_{\infty}$, where $S_{\infty}$ is a large enough sphere in $\mathbb{C}^{n+1}$; see Theorem 3.1 below.

Example 2.8. (i) Let $\mathcal{U}=\left(\mathbb{C}^{*}\right)^{s} \times \mathbb{C}^{n+1-s}$ for some integer $0 \leq s \leq n+1$. Then the universal abelian covering $\mathcal{U}^{a b}$ is contractible, and then $A_{0}(\mathcal{U})=\mathbb{C}_{\mathbf{1}}$ and $A_{k}(\mathcal{U})=0$ for $k>0$. Therefore, by the spectral sequence (2.3) we get $A^{k}(\mathcal{U}) \cong \operatorname{Ext}_{R_{s}}^{k}\left(\mathbb{C}_{1}, R_{s}\right)$ for all $k \geq 0$. Using the free resolution of $\mathbb{C}_{\mathbf{1}}$ given by the Koszul complex of the regular sequence $\left\{x_{j}=t_{j}-1\right\}_{j=1, \ldots, s}$ in the ring $R_{s}$ ([51, Cor. 4.5.5), we obtain that 
$A^{k}(\mathcal{U})=0$ for $k \neq s$ and $A^{s}(\mathcal{U})=\mathbb{C}_{\mathbf{1}}([51]$, Ex. 4.5.2 and Cor 4.5.4). Therefore, for any $\lambda \neq 1$, Proposition 2.3 shows that the corresponding cohomology Alexander modules satisfy $A^{k}(\mathcal{U})_{\lambda}=0$ for any $k$. Moreover $H_{k}\left(\mathcal{U}, \mathcal{L}_{\lambda}\right)=H^{k}\left(\mathcal{U}, \mathcal{L}_{\lambda^{-1}}\right)=0$ for any $k$.

(ii) Let $(Y, 0)$ be an INNC singularity at the origin of $\mathbb{C}^{n+1}$. Set $\mathcal{U}(Y, 0)=$ $B \backslash Y$, where $B$ is a small open ball centered at the origin in $\mathbb{C}^{n+1}$. Assume that $n \geq 2$. Then the universal abelian cover $\mathcal{U}(Y, 0)^{a b}$ of $\mathcal{U}(Y, 0)$ is $(n-1)$ connected; see Libgober [36. More precisely, it is a bouquet of $n$-spheres, see [22], and hence $A_{0}(\mathcal{U}(Y, 0))=\mathbb{C}_{\mathbf{1}}$ and $A_{k}(\mathcal{U}(Y, 0))=0$ for $k \neq n$. As in (i) above, we get $A^{k}(\mathcal{U}(Y, 0)) \cong \operatorname{Ext}_{R_{s}}^{k}\left(\mathbb{C}_{\mathbf{1}}, R_{s}\right)$ for all $k<n$. For $\lambda \neq \mathbf{1}$ this yields $A^{k}(\mathcal{U}(Y, 0))_{\lambda}=0$ for $k<n$, and therefore $H^{k}\left(\mathcal{U}(Y, 0), \mathcal{L}_{\lambda}\right)=0$ for any $k<n$.

\section{Divisibility RESUlts AND CharaCteristic VARIETIES}

In this section we give an algebraic-geometrical interpretation for the multivariable Alexander invariants of the hypersurface complement, similar in flavor to the one-variable case described in 42, but see also the reformulation of these results in 21. We will use an approach based on the general theory of perverse sheaves, close to the one presented in [21] (see also 9] and [18]). Note that the supports and characteristic polynomials $\Delta_{0}$ of the multi-variable Alexander modules are the analogue of the set of roots of the Alexander polynomials and respectively Alexander polynomials in the one-variable case (cf. [42, 21]).

The first result is an extension of [34], Theorem 3.2, to arbitrary hypersurface singularities . Let $S_{\infty}$ be a sphere of sufficiently large radius in $\mathbb{C}^{n+1}=\mathbb{C P}^{n+1} \backslash H$ (or equivalently, the boundary of a sufficiently small tubular neighborhood of $H$ in $\left.\mathbb{C P}^{n+1}\right)$. Let $V_{\infty}=S_{\infty} \cap V$ be the link of $V$ at infinity, and $\mathcal{U}_{\infty}=S_{\infty} \backslash V_{\infty}$ its complement.

Theorem 3.1. For all $i$, and all $k \leq n: V_{i, k}(\mathcal{U}) \subset V_{i, k}\left(\mathcal{U}_{\infty}\right)$, and $\Delta_{i, k}(\mathcal{U}) \mid \Delta_{i, k}\left(\mathcal{U}_{\infty}\right)$. Moreover, for $k<n$, these inclusions and divisibility conditions are replaced by equalities.

Proof. The case $n=1$ is considered in 34. In fact in this situation one sets, for $i \leq 1$ and $k \leq 1, V_{i, k}\left(\mathcal{U}_{\infty}\right)$ to be the $k$-th characteristic variety of the $i$-th homology module of the covering space of $\mathcal{U}_{\infty}$ corresponding to the kernel of the composition

$$
\pi_{1}\left(\mathcal{U}_{\infty}\right) \rightarrow \pi_{1}(\mathcal{U}) \rightarrow H_{1}(\mathcal{U})
$$

For $n \geq 2$, the theorem is an easy consequence of the Lefschetz hyperplane theorem. Indeed, as in the proof of Theorem 4.5 of [32, it follows that $\pi_{1}(\mathcal{U}) \cong \pi_{1}\left(\mathcal{U}_{\infty}\right)$, and more generally $\pi_{k}\left(\mathcal{U}, \mathcal{U}_{\infty}\right) \cong 0$ for all $k \leq n$. Therefore, the same is true for any covering, in particular for the universal abelian coverings: $\pi_{k}\left(\mathcal{U}^{a b}, \mathcal{U}_{\infty}^{a b}\right) \cong 0$ for all $k \leq n$. Hence, by the Hurewicz Theorem, the vanishing also holds for the relative homology groups, i.e., the maps of groups $H_{k}\left(\mathcal{U}_{\infty}^{a b}\right) \rightarrow H_{k}\left(\mathcal{U}^{a b}\right)$ are isomorphisms for $k<n$ and onto for $k=n$. Since these maps are induced by an embedding (recall $n \geq 2$ ), the above are morphisms of modules over the ring of Laurent polynomials in $s$ variables. The statement of the theorem follows now from Lemma 2.1

From now on to the end of this section, we will make the assumption that the hyperplane $H$ at infinity is transversal (in the stratified sense) to the hypersurface $V$. With this assumption, we show that the global cohomological Alexander invariants of the hypersurface complement are entirely determined by the degrees of the 
irreducible components on the one hand, and by the local topological information encoded by the singularities of $V$ on the other hand. In particular, these invariants depend on the local type of singularities of the hypersurface.

First, we need some notation. Recall from $\$ 2.2$ that $A^{q}(\mathcal{U}) \cong H^{q}\left(\mathcal{U}, \mathcal{L}^{\vee}\right)$. For $x \in V$, we let $\mathcal{U}_{x}=\mathcal{U} \cap B_{x}$, for $B_{x}$ a small open ball at $x$ in $\mathbb{C P}^{n+1}$. Denote by $\mathcal{L}_{x}$ the restriction of the local coefficient system $\mathcal{L}$ to $\mathcal{U}_{x}$. Then the groups $H^{*}\left(\mathcal{U}_{x}, \mathcal{L}_{x}^{\vee}\right)$ inherit an $R_{s}$-module structure.

Theorem 3.2. Let $\lambda=\left(\lambda_{1}, \cdots, \lambda_{s}\right) \in \mathbb{T}^{s}$ and $\epsilon \in \mathbb{Z}_{>0}$. Fix an irreducible component $V_{1}$ of $V$, and assume that $\lambda \notin \operatorname{Supp}\left(H^{q}\left(\mathcal{U}_{x}, \mathcal{L}_{x}^{\vee}\right)\right)$ for all $q<n+1-\epsilon$ and all points $x \in V_{1}$. Then $\lambda \notin \operatorname{Supp}\left(A^{q}(\mathcal{U})\right)$ for all $q<n+1-\epsilon$.

Proof. Let $\mathcal{U}_{1}=\mathbb{C P}^{n+1} \backslash V_{1}$, and let $i: \mathcal{U} \hookrightarrow \mathcal{U}_{1}$ and $j: \mathcal{U}_{1} \hookrightarrow \mathbb{C P}^{n+1}$ be the two inclusions. Then $\mathcal{L}^{\vee}[n+1] \in \operatorname{Perv}(\mathcal{U})$, since $\mathcal{U}$ is smooth. Moreover $\mathcal{F}:=R i_{*}\left(\mathcal{L}^{\vee}[n+1]\right) \in \operatorname{Perv}\left(\mathcal{U}_{1}\right)$, since $i$ is a quasi-finite affine morphism (see [48], Theorem 6.0.4). But $\mathcal{U}_{1}$ is affine $(n+1)$-dimensional, and $\mathcal{F} \in \operatorname{Perv}\left(\mathcal{U}_{1}\right)$; therefore by Artin's vanishing theorem for perverse sheaves (see [48], Corollary 6.0.4), the following hold:

$$
\begin{aligned}
& \mathbb{H}^{k}\left(\mathcal{U}_{1}, \mathcal{F}\right)=0, \text { for all } k>0, \\
& \mathbb{H}_{c}^{k}\left(\mathcal{U}_{1}, \mathcal{F}\right)=0, \text { for all } k<0 .
\end{aligned}
$$

Let $a: \mathbb{C P}^{n+1} \rightarrow$ point be the constant map. Then

$$
\mathbb{H}^{k}\left(\mathcal{U}_{1}, \mathcal{F}\right) \cong H^{k+n+1}\left(\mathcal{U}, \mathcal{L}^{\vee}\right) \cong H^{k}\left(R a_{*} R j_{*} \mathcal{F}\right)
$$

and

$$
\mathbb{H}_{c}^{k}\left(\mathcal{U}_{1}, \mathcal{F}\right) \cong H^{k}\left(R a_{!} R j_{!} \mathcal{F}\right)
$$

Note that since $a$ is a proper map, we have $R a_{!}=R a_{*}$.

Now consider the canonical morphism $R j_{!} \mathcal{F} \rightarrow R j_{*} \mathcal{F}$ and extend it to the distinguished triangle

$$
R j_{!} \mathcal{F} \rightarrow R j_{*} \mathcal{F} \rightarrow \mathcal{G} \stackrel{[1]}{\rightarrow}
$$

in $D_{c}^{b}\left(\mathbb{C P}^{n+1}\right)$. Since $j^{*} j_{\text {! }} \cong i d \cong j^{*} j_{*}$, the complex $\mathcal{G}$ is supported on $V_{1}$. Apply $R a_{!}=R a_{*}$ to the above distinguished triangle and obtain

$$
R a_{!} R j ! \mathcal{F} \rightarrow R a_{*} R j_{*} \mathcal{F} \rightarrow R a_{*} \mathcal{G} \stackrel{[1]}{\rightarrow}
$$

Upon applying the cohomology functor to this triangle, and using the above vanishing, we obtain that

$$
H^{k+n+1}\left(\mathcal{U}, \mathcal{L}^{\vee}\right) \cong \mathbb{H}^{k}\left(\mathbb{C P}^{n+1}, \mathcal{G}\right) \cong \mathbb{H}^{k}\left(V_{1}, \mathcal{G}\right) \text { for } k<-1,
$$

and $H^{n}\left(\mathcal{U}, \mathcal{L}^{\vee}\right)$ is a submodule of $\mathbb{H}^{-1}\left(V_{1}, \mathcal{G}\right)$.

Therefore, by Lemma 2.1, in order to prove the theorem it suffices to show that, under our assumptions, $\lambda \notin \operatorname{Supp}\left(\mathbb{H}^{k}\left(V_{1}, \mathcal{G}\right)\right)$ for all $k<-\epsilon$. This follows from the local calculation and the hypercohomology spectral sequence. Indeed, for $x \in V_{1}$, we have

$$
\begin{aligned}
\mathcal{H}^{q}(\mathcal{G})_{x} & \cong \mathcal{H}^{q}\left(R j_{*} \mathcal{F}\right)_{x} \cong \mathcal{H}^{q+n+1}\left(R j_{*} R i_{*} \mathcal{L}^{\vee}\right)_{x} \cong \mathbb{H}^{q+n+1}\left(B_{x}, R(j \circ i)_{*} \mathcal{L}^{\vee}\right) \\
& \cong H^{q+n+1}\left(\mathcal{U}_{x}, \mathcal{L}_{x}^{\vee}\right)
\end{aligned}
$$

where $\mathcal{U}_{x}=\mathcal{U} \cap B_{x}$, for $B_{x}$ a small open ball at $x$ in $\mathbb{C P}^{n+1}$, and $\mathcal{L}_{x}$ is the restriction of the local coefficient system $\mathcal{L}$ to $\mathcal{U}_{x}$. Therefore, for a fixed $x \in V_{1}$ the assumption that $\lambda \notin \operatorname{Supp}\left(H^{q}\left(\mathcal{U}_{x}, \mathcal{L}_{x}^{\vee}\right)\right)$ for all $q<n+1-\epsilon$ is equivalent to the assumption 
$\lambda \notin \operatorname{Supp}\left(\mathcal{H}^{q}(\mathcal{G})_{x}\right)$ for all $q<-\epsilon$. Next note that $\mathbb{H}^{k}\left(V_{1}, \mathcal{G}\right)$ is the abutment of a spectral sequence with the $E_{2}$-term defined by $E_{2}^{p, q}=H^{p}\left(V_{1}, \mathcal{H}^{q}(\mathcal{G})\right)$. Moreover, if $\lambda \notin \operatorname{Supp}\left(\mathcal{H}^{q}(\mathcal{G})_{x}\right)$ for all $q<-\epsilon$ and for all $x \in V_{1}$, then $\lambda \notin \operatorname{Supp}\left(H^{p}\left(V_{1}, \mathcal{H}^{q}(\mathcal{G})\right)\right)$ for $p+q=k<-\epsilon$ (since $E_{2}^{p, q}$ is nontrivial only if $p \geq 0$ ). Thus, from the spectral sequence, it follows that $\lambda \notin \operatorname{Supp}\left(\mathbb{H}^{k}\left(V_{1}, \mathcal{G}\right)\right)$ for all $k<-\epsilon$. This finishes the proof of the theorem.

Remark 3.3. Theorem 3.2 leads to vanishing-type results for the global Alexander invariants of the hypersurface, as a consequence of the vanishing of (supports of) local Alexander invariants at singular points (e.g., see Corollary 3.5 below). In order to derive such a result, we need to clarify the relationship between the local modules $H^{*}\left(\mathcal{U}_{x}, \mathcal{L}_{x}^{\vee}\right)$ that appear in the statement of Theorem 3.2 on the one hand, and the local universal Alexander modules at a singular point $x$ on the other hand. The latter are defined as in 2.2 More precisely, let $\mathcal{U}_{0}$ denote the hypersurface complement $\mathbb{C} \mathbb{P}^{n+1} \backslash V$, and for $x \in V_{1}$ set $\mathcal{U}_{x}^{\prime}=\mathcal{U}_{0} \cap B_{x}$, for $B_{x}$ a small open ball at $x$ in $\mathbb{C P}^{n+1}$. Let $\mathcal{U}_{x}^{a b}$ and $\left(\mathcal{U}_{x}^{\prime}\right)^{a b}$ be the universal abelian covers of $\mathcal{U}_{x}$ and $\mathcal{U}_{x}^{\prime}$, respectively, and denote by $A_{*}\left(\mathcal{U}_{x}\right)$ and respectively $A_{*}\left(\mathcal{U}_{x}^{\prime}\right)$ the associated universal homological Alexander modules. The modules $A_{*}\left(\mathcal{U}_{x}^{\prime}\right)$ are called the local universal homological Alexander modules at $x$, as they depend only on the singularity germ $(V, x)$.

We first relate $H^{*}\left(\mathcal{U}_{x}, \mathcal{L}_{x}^{\vee}\right)$ to the modules $A_{*}\left(\mathcal{U}_{x}\right)$, then express the latter in terms of the local universal Alexander modules at $x$.

If $i_{x}: \mathcal{U}_{x} \hookrightarrow \mathcal{U}$ denotes the inclusion map, then the local system $\mathcal{L}_{x}$ on $\mathcal{U}_{x}$ is induced via the composition of maps

$$
\phi: \pi_{1}\left(\mathcal{U}_{x}\right) \stackrel{\left(i_{x}\right) \#}{\rightarrow} \pi_{1}(\mathcal{U}) \stackrel{\mathrm{lk}}{\rightarrow} H_{1}(\mathcal{U}) \rightarrow \operatorname{Aut}\left(R_{s}\right) .
$$

On the other hand, by the naturality of the Hurewicz morphism, $\phi$ factors through $\mathrm{lk}_{x}: \pi_{1}\left(\mathcal{U}_{x}\right) \rightarrow H_{1}\left(\mathcal{U}_{x}\right), R_{s}$ becoming in this way a (left) $\mathbb{C}\left[H_{1}\left(\mathcal{U}_{x}\right)\right]$-module. Then, by [18], p. 50 , it follows that $H^{*}\left(\mathcal{U}_{x}, \mathcal{L}_{x}^{\vee}\right)$ is the homology of the equivariant Hom:

$$
C^{*}\left(\mathcal{U}_{x}, \mathcal{L}_{x}^{\vee}\right)=\operatorname{Hom}_{\mathbb{C}\left[H_{1}\left(\mathcal{U}_{x}\right)\right]}\left(C_{*}^{0}\left(\mathcal{U}_{x}^{a b}\right), R_{s}\right),
$$

where $R_{s}$ is regarded now as a right $\mathbb{C}\left[H_{1}\left(\mathcal{U}_{x}\right)\right]$-module using the involution on the group ring as in Remark 2.2, and as a left $R_{s}$-module. By [31, p.6, there is a spectral sequence converging to $H^{*}\left(\mathcal{U}_{x}, \mathcal{L}_{x}^{\vee}\right)$ with the $E_{2}$-term given by

$$
E_{2}^{p, q}=\operatorname{Ext}_{\mathbb{C}\left[H_{1}\left(\mathcal{U}_{x}\right)\right]}^{q}\left(A_{p}\left(\mathcal{U}_{x}\right), R_{s}\right)
$$

Thus each module $H^{*}\left(\mathcal{U}_{x}, \mathcal{L}_{x}^{\vee}\right)$ is built up entirely from information carried by the modules $A_{*}\left(\mathcal{U}_{x}\right)$. The latter are related to the local Alexander modules $A_{*}\left(\mathcal{U}_{x}^{\prime}\right)$ by the following observations. For points $x \in V_{1} \backslash\left(V_{1} \cap H\right)$ we have $\mathcal{U}_{x}^{\prime}=\mathcal{U}_{x}$; thus $A_{*}\left(\mathcal{U}_{x}\right)=A_{*}\left(\mathcal{U}_{x}^{\prime}\right)$. For $x \in V_{1} \cap H$, the transversality assumption implies that $\mathcal{U}_{x}$ is homotopy equivalent to $\mathcal{U}_{x}^{\prime} \times S^{1}$. It follows that $\mathcal{U}_{x}^{a b} \simeq\left(\mathcal{U}_{x}^{\prime}\right)^{a b} \times \mathbb{R}$; thus by the homological Künneth formula we obtain that the group $A_{p}\left(\mathcal{U}_{x}\right)$ is isomorphic to $H_{p}\left(\left(\mathcal{U}_{x}^{\prime}\right)^{a b}, \mathbb{C}\right) \otimes H_{0}(\mathbb{R}, \mathbb{C}) \cong A_{p}\left(\mathcal{U}_{x}^{\prime}\right)$. When considering the $\mathbb{C}\left[H_{1}\left(\mathcal{U}_{x}\right)\right]$-module structure, the isomorphism can be written as (see [6], Prop. 1.8):

$$
A_{p}\left(\mathcal{U}_{x}\right) \cong\left(A_{p}\left(\mathcal{U}_{x}^{\prime}\right) \otimes_{\mathbb{C}\left[H_{1}\left(\mathcal{U}_{x}^{\prime}\right)\right]} \mathbb{C}\left[H_{1}\left(\mathcal{U}_{x}\right)\right]\right) \otimes_{\mathbb{C}[\mathbb{Z}]} \mathbb{C} .
$$

Together with the spectral sequence (3.1), this yields the desired relationship.

Remark 3.4. If $S$ is an $s$-dimensional stratum in a Whitney stratification of $V$ such that $x \in S$, then $A_{p}\left(\mathcal{U}_{x}^{\prime}\right)=0$ if $p>n-s$. Indeed, $\mathcal{U}_{x}^{\prime}$ has the homotopy 
type of the link complement $S_{x}^{2 n-2 s+1} \backslash L_{x}$, where $S_{x}^{2 n-2 s+1}$ is a small sphere at $x$ in a submanifold of $\mathbb{C P}^{n+1}$ which meets $S$ transversally at $x$ (and no other point), and $\left(S_{x}^{2 n-2 s+1}, L_{x}\right)$ is the link pair of the stratum $S$ in the pair $\left(\mathbb{C P}^{n+1}, V\right)$. Since $S_{x}^{2 n-2 s+1} \backslash L_{x}$ admits a cyclic cover which has the homotopy type of a CW complex of dimension $n-s$ (i.e., the fiber of the Milnor fibration associated to the algebraic link $\left.\left(S_{x}^{2 n-2 s+1}, L_{x}\right)\right)$, it follows that the universal abelian cover $\left(\mathcal{U}_{x}^{\prime}\right)^{a b}$ has the homotopy type of an $(n-s)$-dimensional CW complex, thus proving the claim.

The following consequence of Theorem 3.2 . Remark 3.3. and of Example 2.8 is similar to some results in 22, 36, 37.

Corollary 3.5. (i) (Case $\epsilon=0$ ) With the notation in the above theorem, assume in addition that $V$ is a normal crossing divisor at any point of the component $V_{1}$. Then $\operatorname{Supp}\left(A^{k}(\mathcal{U})\right) \subset\{\mathbf{1}\}$ for any $k<n+1$.

(ii) (Case $\epsilon=1)$ With the notation in the above theorem, assume in addition that $V$ is an INNC divisor at any point of the component $V_{1}$. Then $\operatorname{Supp}\left(A^{k}(\mathcal{U})\right) \subset\{\mathbf{1}\}$ for any $k<n$.

Using a similar argument (see also 21]) we obtain the following result.

Theorem 3.6. Assume that the hypersurface $V$ is transversal (in the stratified sense) to the hyperplane $H$ at infinity. Then for $k \leq n, \operatorname{Supp}\left(A^{k}(\mathcal{U})\right)$ is contained in the zero set of the polynomial $t_{1}^{d_{1}} \cdots t_{s}^{d_{s}}-1$, thus has positive codimension in $\mathbb{T}^{s}$.

The positive codimension property of supports in the universal abelian case should be regarded as the analogue of the torsion property in the infinite cyclic case (cf. 42, 21]). Example 5.6 below shows that transversality except at finitely many points is not enough to get Theorem 3.6

Proof. As in the proof of the previous theorem, after replacing $\mathcal{U}_{1}$ by the affine space $\mathbb{C}^{n+1}=\mathbb{C P}^{n+1} \backslash H$, it follows that for $k \leq-1, H^{k+n+1}\left(\mathcal{U}, \mathcal{L}^{\vee}\right)$ is a submodule of $\mathbb{H}^{k}\left(\mathbb{C} \mathbb{P}^{n+1}, \mathcal{G}\right)$, where $\mathcal{G}$ is now a complex of sheaves supported on $H$. Therefore, by Lemma 2.1, it suffices to prove the theorem for the supports of the modules $\mathbb{H}^{k}(H, \mathcal{G})$ with $k \leq-1$.

As in the previous theorem, for $x \in H$, the local calculation on stalks yields $\mathcal{H}^{q}(\mathcal{G})_{x} \cong H^{q+n+1}\left(\mathcal{U}_{x}, \mathcal{L}_{x}^{\vee}\right)$, where $\mathcal{U}_{x}=\mathcal{U} \cap B_{x}$, for $B_{x}$ a small open ball at $x$ in $\mathbb{C P}^{n+1}$. If $x \in H \backslash H \cap V$, then $\mathcal{U}_{x}$ is homotopy equivalent to $\mathbb{C}^{*}$, and the corresponding local system $\mathcal{L}_{x}^{\vee}$ is defined by the action of $\gamma_{\infty}$, i.e., by multiplication by $\prod_{j=1}^{s}\left(t_{j}\right)^{d_{j}}$. On the other hand, if $x \in V \cap H$, then due to the transversality assumption, $\mathcal{U}_{x}$ is homotopy equivalent to a product $\left(B_{x}^{\prime} \backslash V \cap B_{x}^{\prime}\right) \times \mathbb{C}^{*}$, with $B_{x}^{\prime}$ a small open ball centered at $x$ in $H$, and the local system $\mathcal{L}_{x}^{\vee}$ is an external tensor product, the second factor being defined by multiplication by $\prod_{j=1}^{s}\left(t_{j}\right)^{d_{j}}$. Thus, by the Kunneth spectral sequence, the stalk cohomology groups of $\mathcal{G}$ along $H$, i.e. $\mathcal{H}^{q}(\mathcal{G})_{x \in H}$, have supports contained in the zero set of the polynomial $t_{1}^{d_{1}} \cdots t_{s}^{d_{s}}-1$. Then by the hypercohomology spectral sequence, the same is true for the supports of the hypercohomology groups $\mathbb{H}^{k}(H, \mathcal{G})$.

\section{Explicit COMPUTATIONS VIA LOGARITHMiC CONNECTIONS}

We review a general method used to determine the characteristic varieties in the case of hyperplane arrangements, see [25] and [47, and show that essentially the same method applies to more general situations as well. 
Let $\pi:(Z, D) \rightarrow\left(\mathbb{C P}^{n+1}, V \cup H\right)$ be an embedded resolution of singularities for the reduced divisor $V \cup H$. In particular,

(i) $D$ is a normal crossing divisor with smooth irreducible components;

(ii) $\pi: Z \backslash D \rightarrow \mathcal{U}$ is an isomorphism.

In this setting there is a Hodge-Deligne spectral sequence

$$
E_{1}^{p, q}=H^{q}\left(Z, \Omega_{Z}^{p}(\log D)\right) \Rightarrow H^{p+q}(\mathcal{U}, \mathbb{C})
$$

degenerating at $E_{1}$ and inducing the Hodge filtration $F$ of the Deligne mixed Hodge structure on $H^{p+q}(\mathcal{U}, \mathbb{C})$; see [13].

Corollary 4.1. If the Deligne mixed Hodge structure on some cohomology space $H^{m}(\mathcal{U})$ is pure of type $(m, m)$, then

(i) $H^{0}\left(Z, \Omega_{Z}^{m}(\log D)\right)=H^{m}(\mathcal{U})$ and

(ii) $H^{q}\left(Z, \Omega_{Z}^{p}(\log D)\right)=0$ for $p+q=m$ and $q>0$.

We list below several cases when this property holds.

Example 4.2. (a) When $V$ is a hyperplane arrangement, the cohomology space $H^{m}(\mathcal{U})$ is pure of type $(m, m)$ for all $m \geq 0$; see [19].

(b) When $V$ is a smooth rational curve arrangement in the projective plane (i.e., any irreducible component of $V$ is either a line or a smooth conic), the cohomology space $H^{m}(\mathcal{U})$ is pure of type $(m, m)$ for all $m \geq 0$ (easy exercise for the reader).

(c) $H^{m}(\mathcal{U})$ is always pure of type $(m, m)$ for all $m \leq 1$. This follows from the fact that $g=\left(g_{1}, \ldots, g_{s}\right): \mathcal{U} \rightarrow \mathbb{T}^{s}$ induces an isomorphism at the $H^{m}$-level for all $m \leq 1$. Here we look at $\mathcal{U}$ as a subset of $\mathbb{C}^{n+1}$ and we set $g_{j}\left(x_{1}, \ldots, x_{n+1}\right)=$ $f_{j}\left(1, x_{1}, \ldots, x_{n+1}\right)$.

For $\lambda=\left(\lambda_{1}, \ldots, \lambda_{s}\right) \in \mathbb{T}^{s}$, let $\mathcal{L}_{\lambda}$ be the corresponding local system on $\mathcal{U}=Z \backslash D$. Let $\alpha_{j} \in \mathbb{C}$ be such that $\exp \left(-2 \pi i \alpha_{j}\right)=\lambda_{j}$ for $j=1, \ldots, s$. Then $\mathcal{L}_{\lambda}$ is the local system of horizontal sections of the connection

$$
\nabla_{\alpha}: \mathcal{O}_{\mathcal{U}} \rightarrow \Omega_{\mathcal{U}}^{1}
$$

given by $\nabla_{\alpha}(u)=d u+u \cdot \omega_{\alpha}$, where

$$
\omega_{\alpha}=\sum_{j=1, s} \alpha_{j} \frac{d g_{j}}{g_{j}} .
$$

Alternatively, if we look at $\mathcal{U}$ as a subset of $\mathbb{C P}^{n+1}$, then we can use the formula

$$
\omega_{\alpha}=\sum_{j=0, s} \alpha_{j} \frac{d f_{j}}{f_{j}}
$$

where we set $\alpha_{0}=-\sum_{j=1, s} d_{j} \cdot \alpha_{j}$. Recall that $f_{0}=x_{0}$.

Using the fact that $\mathcal{U}$ is affine and our connection is regular, it follows that

$$
H^{m}\left(\mathcal{U}, \mathcal{L}_{\lambda}\right)=H^{m}\left(H^{0}\left(\mathcal{U}, \Omega_{\mathcal{U}}^{*}\right), \nabla_{\alpha}\right)
$$

just as in [18, Thm. 3.4.18] or, for complete proofs, 12. However, this result is not so useful to perform explicit computations since the groups $H^{0}\left(\mathcal{U}, \Omega_{\mathcal{U}}^{*}\right)$ are too large.

There is a second approach to computing $H^{m}\left(\mathcal{U}, \mathcal{L}_{\lambda}\right)$, this time using logarithmic connections. It has the advantage of reducing the size of the spaces $H^{0}\left(\mathcal{U}, \Omega_{\mathcal{U}}^{*}\right)$, but one has to be more careful about the residues $\alpha_{j}$. More precisely, the pull-back of the connection $\nabla_{\alpha}$ under the embedded resolution $\pi$ is a logarithmic connection 
$\tilde{\nabla}_{\alpha}$ on $Z$ with poles along $D$. Let $\rho_{i}$ be the residue of the connection $\tilde{\nabla}_{\alpha}$ along the irreducible component $D_{i}$ of $D$. When $D_{i}$ is the proper transform of some component $V_{j}$ of $V$ one has $\rho_{i}=\alpha_{j}$.

Definition 4.3. A choice of residues $\alpha=\left(\alpha_{0}, \alpha_{1}, \ldots, \alpha_{s}\right)$ for $\mathcal{L}_{\lambda}$ as above is an admissible choice of residues for $\mathcal{L}_{\lambda}$ if $\rho_{i} \notin \mathbb{N}_{>0}$ for all irreducible components $D_{i}$ of $D$. A rank one local system $\mathcal{L}_{\lambda}$ is admissible if there is some admissible choice of residues for it.

Remark 4.4. It is easy to see, using Hironaka's embedded resolution of singularities and by blowing-up smooth subvarieties, that for any $i$ there is a relation

$$
\rho_{i}=\sum_{j=1, s} n_{i j} \alpha_{j}
$$

with $n_{i j} \in \mathbb{Z}$ (see [25] for similar formulas and note that negative coefficients occur due to the presence of the hyperplane at infinity). The condition $\rho_{i} \notin \mathbb{N}_{>0}$ is clearly satisfied if all $\alpha_{j}$ are sufficiently small. In other words, there is a neighborhood $U(\mathbf{1})$ of the trivial local system $1 \in \mathbb{T}^{s}$ formed entirely by admissible local systems.

If we move away from the trivial local system, it is not clear whether all the local systems are admissible. The answer to this question is negative for some hyperplane arrangements; see [7, Example 4.4], [5. Example 3.4], 38] and [49. On the other hand, for not very complicated arrangements, see Examples 4.8 and 4.10 below, the answer is positive.

For an admissible choice of residues one has an $E_{1}$-spectral sequence

$$
E_{1}^{p, q}=H^{q}\left(Z, \Omega_{Z}^{p}(\log D)\right) \Rightarrow H^{p+q}\left(\mathcal{U}, \mathcal{L}_{\lambda}\right)
$$

whose differential $d_{1}$ is induced by $\tilde{\nabla}_{\alpha}$; see [18, Thm. 3.4.11 (i)]. The above discussion proves the following.

Proposition 4.5. Assume that $\alpha=\left(\alpha_{0}, \alpha_{1}, \ldots, \alpha_{s}\right)$ is an admissible choice of residues for $\mathcal{L}_{\lambda}$ and that the cohomology groups $H^{m}(\mathcal{U})$ are pure of type $(m, m)$ for all $m \leq k$. Then

$$
H^{m}\left(\mathcal{U}, \mathcal{L}_{\lambda}\right)=H^{m}\left(H^{*}(\mathcal{U}), \omega_{\alpha} \wedge\right)
$$

for all $m \leq k$ and $H^{k+1}\left(H^{*}(\mathcal{U}), \omega_{\alpha} \wedge\right)$ is a subspace in $H^{k+1}\left(\mathcal{U}, \mathcal{L}_{\lambda}\right)$.

When $\mathcal{U}$ is a hyperplane arrangement complement, this is exactly the argument used in 25] and [4]. Proposition 4.5, Remark 4.4 and Example 4.2 yield the following.

Corollary 4.6. If $\mathcal{U}$ is an affine hypersurface arrangement complement, then there is a neighborhood $U(\mathbf{1})$ of the trivial local system $\mathbf{1} \in \mathbb{T}^{s}$ such that

$$
H^{1}\left(\mathcal{U}, \mathcal{L}_{\lambda}\right)=H^{1}\left(H^{*}(\mathcal{U}), \omega_{\alpha} \wedge\right)
$$

for any local system $\mathcal{L}_{\lambda} \in U(\mathbf{1}), \alpha$ being an arbitrary choice of admissible residues for $\mathcal{L}_{\lambda}$.

Corollary 4.7. If $\mathcal{U}=M(\mathcal{A})$ is a hyperplane arrangement complement, then there is a neighborhood $U(\mathbf{1})$ of the trivial local system $\mathbf{1} \in \mathbb{T}^{s}$ such that

$$
H^{m}\left(\mathcal{U}, \mathcal{L}_{\lambda}\right)=H^{m}\left(H^{*}(\mathcal{U}), \omega_{\alpha} \wedge\right)
$$

for any $m \in \mathbb{N}$, and any local system $\mathcal{L}_{\lambda} \in U(\mathbf{1})$, $\alpha$ being an arbitrary choice of admissible residues for $\mathcal{L}_{\lambda}$. 
In relation to the isomorphism (4.5), we note that in the case of hyperplane arrangement complements the following inequality holds for any $m \in \mathbb{N}$ and any character $\lambda \in \mathbb{T}^{s}$ ([38], Proposition 4.2):

$$
\operatorname{dim} H^{m}\left(\mathcal{U}, \mathcal{L}_{\lambda}\right) \geq \operatorname{dim} H^{m}\left(H^{*}(\mathcal{U}), \omega_{\alpha} \wedge\right) .
$$

However, the opposite inequality is false in general (see [49], Example 4.1).

Example 4.8. In the projective plane $\mathbb{C P}^{2}$ consider the hypersurface $V$ having as irreducible components $V_{1}: x=0, V_{2}: y=0, V_{3}: x^{2}-y z=0$. Let $H=V_{0}$ be the line at infinity given by $z=0$ and note that $H$ is not transverse in a stratified sense to $V$. Consider the connection $\nabla_{\lambda}$ whose residues are $\alpha=\left(\alpha_{0}, \alpha_{1}, \ldots, \alpha_{3}\right)$ with

$$
\alpha_{0}=-\alpha_{1}-\alpha_{2}-2 \alpha_{3} \text {. }
$$

Let $A=V_{1} \cap V_{2} \cap V_{3}=(0: 0: 1)$ and $B=V_{1} \cap V_{0} \cap V_{3}=(0: 1: 0)$. To construct the embedded resolution of $V \cup H$ we first blow up the points $A$ and $B$, creating thus two exceptional divisors, $D_{A}$ and respectively $D_{B}$. The corresponding residues along $D_{A}$ and $D_{B}$ are easily computable and we get $\alpha_{A}=\alpha_{1}+\alpha_{2}+\alpha_{3}$ and respectively $\alpha_{B}=\alpha_{1}+\alpha_{0}+\alpha_{3}=-\alpha_{2}-\alpha_{3}$. Let $P=D_{A} \cap V_{2}^{\prime} \cap V_{3}^{\prime}$ and $Q=D_{B} \cap V_{0}^{\prime} \cap V_{3}^{\prime}$, where' denotes the proper transform of a divisor. To get the embedded resolution of $V \cup H$ we just have to blow up the points $P$ and $Q$, creating thus two new exceptional divisors, $D_{P}$ and respectively $D_{Q}$. The corresponding residues are $\alpha_{P}=-\alpha_{Q}=\alpha_{1}+2 \alpha_{2}+2 \alpha_{3}$. Therefore the choice of residues $\alpha=\left(\alpha_{0}, \alpha_{1}, \ldots, \alpha_{3}\right)$ is admissible if and only if none of the residues

$\alpha_{1}, \alpha_{2}, \alpha_{3},-\alpha_{1}-\alpha_{2}-2 \alpha_{3}, \alpha_{1}+\alpha_{2}+\alpha_{3},-\alpha_{2}-\alpha_{3}, \alpha_{1}+2 \alpha_{2}+2 \alpha_{3},-\left(\alpha_{1}+2 \alpha_{2}+2 \alpha_{3}\right)$ is a strictly positive integer.

Lemma 4.9. In the situation of Example 4.8, any rank one local system is admissible.

Proof. It is clearly enough to consider the case of real residues $\alpha_{j}$. Otherwise, we just look at the corresponding real parts.

We divide the possibilities into the following two cases.

Case 1. $\left(\alpha_{1}+2 \alpha_{2}+2 \alpha_{3} \notin \mathbb{Z}\right)$.

Suppose first that, in addition, $\alpha_{1}+\alpha_{2}+\alpha_{3} \notin \mathbb{Z}$. Then the choice with $\alpha_{j} \in[0,1)$ for $j=1,2,3$ is admissible.

Now suppose that $\alpha_{1}+\alpha_{2}+\alpha_{3} \in \mathbb{Z}$. It follows that $\alpha_{2}+\alpha_{3} \notin \mathbb{Z}$. Then the choice with $\alpha_{j} \in[0,1)$ for $j=2,3$ and $\alpha_{1}<0$ such that $\alpha_{1}+\alpha_{2}+\alpha_{3}=0$ is admissible.

Case 2. $\left(\alpha_{1}+2 \alpha_{2}+2 \alpha_{3} \in \mathbb{Z}\right)$.

Then we have to choose $\alpha_{1}=-2 \alpha_{2}-2 \alpha_{3}$. The residues in this case are just

$$
-2\left(\alpha_{2}+\alpha_{3}\right),-\left(\alpha_{2}+\alpha_{3}\right), \alpha_{2}, \alpha_{3} .
$$

Hence it is enough to take $\alpha_{j} \in[0,1)$ for $j=2,3$.

Now we continue Example 4.8 by applying Example 4.2 and Proposition 4.5 to get $H^{m}\left(\mathcal{U}, \mathcal{L}_{\lambda}\right)=H^{m}\left(H^{*}(\mathcal{U}), \omega_{\alpha} \wedge\right)$ for all $m$. In order to perform this computation, we need a precise description of the cohomology algebra $H^{*}(\mathcal{U})$ (with $\mathbb{C}$ coefficients), and this can be obtained in this example from the local considerations in [16, pp. 47-49]. The result can be described as follows:

(i) $H^{0}(\mathcal{U})=\mathbb{C}$ and the generator is 1 ;

(ii) $H^{1}(\mathcal{U})=\mathbb{C}^{3}$ and a basis is given by $\eta_{1}=\frac{d x}{x}, \eta_{2}=\frac{d y}{y}$ and $\eta_{3}=\frac{d\left(x^{2}-y\right)}{x^{2}-y}$; 
(iii) $H^{2}(\mathcal{U})=\mathbb{C}^{2}$ and a basis is given by $\eta_{12}=\eta_{1} \wedge \eta_{2}$ and $\eta_{23}=\eta_{2} \wedge \eta_{3}$. The multiplication is given by the relation

$$
2 \eta_{1} \wedge \eta_{3}=2 \eta_{12}+\eta_{23}
$$

(iv) Since $\mathcal{U}$ is affine, $H^{m}(\mathcal{U})=0$ for $m>2$.

The computation of $H^{m}\left(H^{*}(\mathcal{U}), \omega_{\lambda} \wedge\right)$ falls into 3 cases.

Case 1. $\alpha_{1}=\alpha_{2}=\alpha_{3}=0$ and $\mathcal{L}_{\lambda}=\mathbb{C}$ is the constant local system. Then of course $H^{m}\left(\mathcal{U}, \mathcal{L}_{\lambda}\right)=H^{m}(\mathcal{U})$ for all $m$.

Case 2. $\alpha_{1}+2 \alpha_{2}+2 \alpha_{3}=0$. Then a direct computation shows that $H^{0}\left(\mathcal{U}, \mathcal{L}_{\lambda}\right)=0$ and $\operatorname{dim} H^{1}\left(\mathcal{U}, \mathcal{L}_{\lambda}\right)=\operatorname{dim} H^{2}\left(\mathcal{U}, \mathcal{L}_{\lambda}\right)=1$.

Case 3. $\alpha_{1}+2 \alpha_{2}+2 \alpha_{3} \neq 0$. Again a direct computation shows that $H^{0}\left(\mathcal{U}, \mathcal{L}_{\lambda}\right)=$ $H^{1}\left(\mathcal{U}, \mathcal{L}_{\lambda}\right)=H^{2}\left(\mathcal{U}, \mathcal{L}_{\lambda}\right)=0$.

The above computations yield the following equalities.

$$
\begin{gathered}
V_{t}^{0,1}(\mathcal{U})=V_{t}^{0,2}(\mathcal{U})=\left\{\lambda \in \mathbb{T}^{3} ; \quad \lambda_{1} \lambda_{2}^{2} \lambda_{3}^{2}=1\right\} \\
V_{t}^{1,1}(\mathcal{U})=V_{t}^{2,1}(\mathcal{U})=V_{t}^{1,2}(\mathcal{U})=\{\mathbf{1}\}
\end{gathered}
$$

$V_{t}^{m, 1}(\mathcal{U})=\emptyset$ for $m>2$ and $V_{t}^{m, 2}(\mathcal{U})=\emptyset$ for $m>1$.

These results are consistent with the general results by Arapura [1. See also Suciu [9] for a related discussion.

Note that the above 2-dimensional subtorus $\mathbb{T}=\left\{\lambda \in \mathbb{T}^{3} ; \quad \lambda_{1} \lambda_{2}^{2} \lambda_{3}^{2}=1\right\}$ is different from the 2-dimensional subtorus predicted by Theorem 3.6 in the case of a divisor $V$ transverse to the line at infinity.

A special class of local systems is formed by the equimonodromical local systems $\mathcal{L}_{\lambda}$ such that $\lambda_{0}=\lambda_{1}=\ldots=\lambda_{3}$. Then, for $\lambda_{0}^{5}=1$, the dimension of the cohomology space $H^{m}\left(\mathcal{U}, \mathcal{L}_{\lambda}\right)$ is exactly the multiplicity of the root $t=\lambda_{0}$ in the characteristic polynomial

$$
\Delta^{m}(t)=\operatorname{det}\left(t \cdot I d-h^{m}\right),
$$

where $F: x y z\left(x^{2}-y z\right)=1$ is the associated Milnor fiber of $x y z\left(x^{2}-y z\right)$ in $\mathbb{C}^{3}$ and $h: F \rightarrow F$ is the monodromy operator; see for instance [18, 6.4.6]. To compute the cohomology of such an equimonodromical local system $\mathcal{L}_{\lambda}$, one should start by an admissible choice for the residues $\alpha=\left(\alpha_{0}, \alpha_{1}, \alpha_{2}, \alpha_{3}\right)$. For instance, the obvious choice $\alpha=\left(\frac{-4}{5}, \frac{1}{5}, \frac{1}{5}, \frac{1}{5}\right)$ is not admissible. A good choice here is $\alpha=\left(\frac{1}{5}, \frac{-4}{5}, \frac{1}{5}, \frac{1}{5}\right)$. Using this choice, we get the following characteristic polynomials in this situation:

$$
\Delta^{0}(t)=t-1, \Delta^{1}(t)=(t-1)^{2}\left(t^{5}-1\right), \Delta^{2}(t)=(t-1)\left(t^{5}-1\right) .
$$

The following example is similar to the previous one, but it exhibits a curve $V$ which is transversal to the line $H$ at infinity and it needs a different approach for the computation of the cohomology algebra $H^{*}(\mathcal{U})$. Moreover, in this case the cohomology algebra $H^{*}(\mathcal{U})$ is not spanned by the degree one part $H^{1}(\mathcal{U})$.

Example 4.10. In the projective plane $\mathbb{C P}^{2}$ consider the hypersurface $V$ having as irreducible components $V_{1}: x=0, V_{2}: y=0, V_{3}: x^{2}-y^{2}+y z=0$. Let $H=V_{0}$ be the line at infinity given by $z=0$ and note that $H$ is transverse in a stratified sense to $V$ (i.e., each irreducible component of $V$ is smooth, $H$ is transverse to each of them and avoids the intersection points). Consider the connection $\nabla_{\lambda}$ whose residues are $\alpha=\left(\alpha_{0}, \alpha_{1}, \ldots, \alpha_{3}\right)$ with

$$
\alpha_{0}=-\alpha_{1}-\alpha_{2}-2 \alpha_{3}
$$


Let $A=V_{1} \cap V_{2} \cap V_{3}=(0: 0: 1)$. To construct the embedded resolution of $V \cup H$ we first blow up the point $A$, creating an exceptional divisor $D_{A}$. The corresponding residue along $D_{A}$ is $\alpha_{A}=\alpha_{1}+\alpha_{2}+\alpha_{3}$. Let $P=D_{A} \cap V_{2}^{\prime} \cap V_{3}^{\prime}$, where ' denotes the proper transform of a divisor. To get the embedded resolution of $V \cup H$ we just have to blow up the point $P$, creating a new exceptional divisor $D_{P}$. The corresponding residue is $\alpha_{P}=\alpha_{1}+2 \alpha_{2}+2 \alpha_{3}$. Therefore the choice of residues $\alpha=\left(\alpha_{0}, \alpha_{1}, \ldots, \alpha_{3}\right)$ is admissible in this case if and only if none of the residues

$$
\alpha_{1}, \alpha_{2}, \alpha_{3},-\alpha_{1}-\alpha_{2}-2 \alpha_{3}, \alpha_{1}+\alpha_{2}+\alpha_{3}, \alpha_{1}+2 \alpha_{2}+2 \alpha_{3}
$$

is a strictly positive integer. It can be shown, exactly as in Lemma 4.9 above, that in this situation any rank one local system is admissible.

It follows that we can apply Example 4.2 and Proposition 4.5 to get $H^{m}\left(\mathcal{U}, \mathcal{L}_{\lambda}\right)=$ $H^{m}\left(H^{*}(\mathcal{U}), \omega_{\lambda} \wedge\right)$ for all $m$. To get a precise description of the cohomology algebra $H^{*}(\mathcal{U})$ we can proceed as follows.

(i) $H^{0}(\mathcal{U})=\mathbb{C}$ and the generator is 1 ;

(ii) $H^{1}(\mathcal{U})=\mathbb{C}^{3}$ and a basis is given by $\eta_{1}=\frac{d x}{x}, \eta_{2}=\frac{d y}{y}$ and $\eta_{3}=\frac{d\left(x^{2}-y^{2}+y\right)}{x^{2}-y^{2}+y}$;

(iii) To compute $H^{2}(\mathcal{U})$ is the first difficulty. This can be done by setting $\mathcal{U}^{0}=$ $\mathbb{C P}^{2} \backslash\left(V_{0} \cup V_{1} \cup V_{2}\right), V_{3}^{0}=V_{3} \backslash\left(V_{0} \cup V_{1} \cup V_{2}\right)$ and considering the Gysin sequence

$$
H^{1}(\mathcal{U}) \rightarrow H^{0}\left(V_{3}^{0}\right) \rightarrow H^{2}\left(\mathcal{U}^{0}\right) \rightarrow H^{2}(\mathcal{U}) \rightarrow H^{1}\left(V_{3}^{0}\right) \rightarrow 0
$$

The first morphism, given by the Poincaré-Leray residue $R$, is clearly surjective, i.e. $R\left(\eta_{3}\right)=1$. Then $\operatorname{dim} H^{2}\left(\mathcal{U}^{0}\right)=1$ and a generator is $\eta_{12}=\eta_{1} \wedge \eta_{2}$. The affine curve $V_{3}^{0}$ is isomorphic to $\mathbb{C} \backslash\{-1,0,1\}$ under the parametrization

$$
x=\frac{t}{t^{2}-1}, y=\frac{t^{2}}{t^{2}-1} .
$$

Using this parametrization, we can identify $H^{1}\left(V_{3}^{0}\right)$ with $\mathbb{C}^{3}$ by sending a rational differential form to its residues at the points $\{-1,0,1\}$. Some explicit computations involving the last nonzero morphism in the exact sequence above (which is again given by the Poincaré-Leray residue $R$ ) show that $R\left(\eta_{13}\right)$ and $R\left(\eta_{23}\right)$ are linearly independent in $H^{1}\left(V_{3}^{0}\right)=\mathbb{C}^{3}$, where $\eta_{13}=\eta_{1} \wedge \eta_{3}$ and $\eta_{23}=\eta_{2} \wedge \eta_{3}$. It follows that $\eta_{12}, \eta_{13}$ and $\eta_{23}$ are linearly independent in $H^{2}(\mathcal{U})$, which is 4-dimensional.

It follows that the following cases are possible in this example.

Case 1. $\alpha_{1}=\alpha_{2}=\alpha_{3}=0$ and $\mathcal{L}_{\lambda}=\mathbb{C}$ is the constant local system. Then of course $H^{m}\left(\mathcal{U}, \mathcal{L}_{\lambda}\right)=H^{m}(\mathcal{U})$ for all $m$.

Case 2. $\left(\alpha_{1}, \alpha_{2}, \alpha_{3}\right) \neq(0,0,0)$. Then a direct computation shows that $H^{0}\left(\mathcal{U}, \mathcal{L}_{\lambda}\right)$ $=H^{1}\left(\mathcal{U}, \mathcal{L}_{\lambda}\right)=0$ and $\operatorname{dim} H^{2}\left(\mathcal{U}, \mathcal{L}_{\lambda}\right)=2$.

The above computations yield the following equalities:

$$
V_{t}^{0,2}(\mathcal{U})=V_{t}^{1,2}(\mathcal{U})=\mathbb{T}^{3}
$$

(hence here the support has 0 codimension),

$$
V_{t}^{1,1}(\mathcal{U})=V_{t}^{2,1}(\mathcal{U})=V_{t}^{2,2}(\mathcal{U})=V_{t}^{3,2}(\mathcal{U})=\{\mathbf{1}\}
$$

$V_{t}^{m, 1}(\mathcal{U})=\emptyset$ for $m>2$ and $V_{t}^{m, 2}(\mathcal{U})=\emptyset$ for $m>3$. Note that the inclusion in Theorem 3.6 is strict in this case.

Consider as in the above example the associated Milnor fiber $F: x y z\left(x^{2}-y^{2}+\right.$ $y z)=1$ and the monodromy operator $h: F \rightarrow F$. A good choice of residue is again 
given by $\alpha=\left(\frac{1}{5}, \frac{-4}{5}, \frac{1}{5}, \frac{1}{5}\right)$. Using this choice, we get the following characteristic polynomials in this situation:

$$
\Delta^{0}(t)=t-1, \Delta^{1}(t)=(t-1)^{3}, \Delta^{2}(t)=(t-1)^{2}\left(t^{5}-1\right)^{2} .
$$

Remark 4.11. In order to apply Theorem 3.2, we have to check the vanishing of some local cohomology groups. When the hypersurface germs occurring in these local complements are quasi-homogeneous, then we can globalize the local situation and compute the corresponding local cohomology groups using the ideas explained in this section. For instance, Example 4.8 covers the case of a plane curve singularity consisting of 3 smooth branches $\left(C_{1}, 0\right),\left(C_{2}, 0\right)$ and $\left(C_{3}, 0\right)$ such that the intersection multiplicities are given by $\left(C_{1}, C_{2}\right)=1,\left(C_{1}, C_{3}\right)=1$ and $\left(C_{2}, C_{3}\right)=2$. This follows from the topological classification of the plane curve germs; see [16, p. $45]$.

\section{A MORE GENERAL SETting}

In this section we define multi-variable Alexander invariants in a more general setting (see below) and attempt to relate them to the invariants previously defined.

Assume that the hypersurface $V$ in $\mathbb{C P}^{n+1}$ has $s$ irreducible components $V_{i}$ with degrees $\operatorname{deg}\left(V_{i}\right)=d_{i}$ for $i=1, \cdots, s$. Denote by $\mathcal{U}_{0}$ the complement $\mathbb{C P}^{n+1} \backslash V$, and let $d=$ g.c.d. $\left(d_{1}, \cdots, d_{s}\right)$. Then

$$
H_{1}\left(\mathcal{U}_{0}\right)=\mathbb{Z}^{s-1} \oplus(\mathbb{Z} / d \mathbb{Z})
$$

is generated by the meridians $\gamma_{i}$ about the nonsingular part of each component $V_{i}$, for $i=1, \cdots, s$ (cf. [16, (4.1.3)). These meridians satisfy a single relation, namely,

$$
\sum_{i=1}^{s} d_{i} \gamma_{i}=0
$$

Now fix a hyperplane $H$ and set, as before, $\mathcal{U}=\mathbb{C P}^{n+1} \backslash(V \cup H)$. Recall that $H_{1}(\mathcal{U})=\mathbb{Z}^{s}$, freely generated by the meridians $\gamma_{i}, i=1, \cdots, s$. Let $i: \mathcal{U} \hookrightarrow \mathcal{U}_{0}$ be the inclusion map, and denote by $\mathcal{U}_{0}^{a b}$ and $\mathcal{U}^{a b}$ the universal abelian covers of $\mathcal{U}_{0}$ and $\mathcal{U}$ respectively, and by $p_{0}$ and $p$ the corresponding covering projections.

The invariants we are interested in are those associated with $\mathcal{U}_{0}^{a b}$, and they are regarded as modules over the quotient ring

$$
\mathbb{C}\left[H_{1}\left(\mathcal{U}_{0}\right)\right]=\mathbb{C}\left[t_{1}^{ \pm 1}, \cdots, t_{s}^{ \pm 1}\right] /\left(t_{1}^{d_{1}} \cdots t_{s}^{d_{s}}-1\right) .
$$

It is a natural question to find the relation between the universal abelian invariants associated with the complement of $V$, and those associated with the complement of $V \cup H$.

For a topological space $X$, let $\mathcal{L}(X)$ denote the set of rank one complex local systems on $X$. When $X=\mathcal{U}$, then $\mathcal{L}(\mathcal{U})$ is naturally identified with the $s$-dimensional complex torus $\mathbb{T}^{s}$. For $X=\mathcal{U}_{0}$, the set $\mathcal{L}\left(\mathcal{U}_{0}\right)$ corresponds to the subset in $\mathbb{T}^{s}$ given by

$$
\left\{\lambda=\left(\lambda_{1}, \ldots, \lambda_{s}\right) \in \mathbb{T}^{s} \mid \lambda_{1}^{d_{1}} \cdots \lambda_{s}^{d_{s}}=1\right\}
$$

With the notation above, let $d_{j}=d \cdot d_{j}^{\prime}$ and consider the $(s-1)$-dimensional complex subtorus

$$
\mathbb{T}=\left\{\lambda=\left(\lambda_{1}, \ldots, \lambda_{s}\right) \in \mathbb{T}^{s} \mid \lambda_{1}^{d_{1}^{\prime}} \cdots \lambda_{s}^{d_{s}^{\prime}}=1\right\} .
$$


For each $d$-root of unity $\beta$, let $\lambda(\beta)$ be one point in the hypersurface in $\mathbb{T}^{s}$ given by the equation

$$
\lambda_{1}^{d_{1}^{\prime}} \cdots \lambda_{s}^{d_{s}^{\prime}}=\beta
$$

Then $\mathcal{L}\left(\mathcal{U}_{0}\right)$ is precisely the disjoint union of translated tori given by

$$
\mathcal{L}\left(\mathcal{U}_{0}\right)=\bigcup_{\beta} \lambda(\beta) \mathbb{T}
$$

The discussion in the previous section relating local systems to connections can be extended to this setting in an obvious way. For instance, we should now use the 1 -form

$$
\omega_{\alpha}=\sum_{j=1, s} \alpha_{j} \frac{d f_{j}}{f_{j}},
$$

where the residues $\alpha$ satisfy the condition $\sum_{j=1, s} d_{j} \cdot \alpha_{j}=0$, which is a necessary condition in order to have a 1 -form on $\mathcal{U}_{0}$.

A different way of looking at a local system $\mathcal{L}$ in $\mathcal{L}\left(\mathcal{U}_{0}\right)$ is by considering it as a local system in $\mathcal{L}(\mathcal{U})$ (given by the obvious restriction $\mathcal{L} \mid \mathcal{U}$ ) such that the action of the elementary loop about the hyperplane $H$ is trivial. This viewpoint yields the following exact sequence:

$$
\cdots \rightarrow H^{k}\left(\mathcal{U}_{0}, \mathcal{L}\right) \rightarrow H^{k}(\mathcal{U}, \mathcal{L}) \rightarrow H^{k-1}\left(\mathcal{U}_{0} \cap H, \mathcal{L}\right) \rightarrow H^{k+1}\left(\mathcal{U}_{0}, \mathcal{L}\right) \rightarrow \cdots
$$

for details on this see [18, pp. 221-222]. The following consequence should be compared to [46, 39, Proposition 1.3]. The higher-dimensional case, but with a generic hyperplane $H$ at infinity, was considered in [32, Lemmas 1.5, 1.11 and 1.13].

Corollary 5.1. Assume that $V$ is a plane curve arrangement, i.e. $n=1$. Then, for any rank one local system $\mathcal{L}=\mathcal{L}_{\lambda}$ on $\mathcal{U}_{0}$ and any choice of the line $H$ at infinity, one has

$$
\operatorname{dim} H^{1}(\mathcal{U}, \mathcal{L})=\operatorname{dim} H^{1}\left(\mathcal{U}_{0}, \mathcal{L}\right)+\epsilon .
$$

Here $\epsilon \in\{0,1\}$ and $\epsilon=0$ if there is a point $p \in V \cap H$ such that

$$
\prod_{j=1, s} \lambda_{j}^{k_{j}} \neq 1
$$

where $k_{j}=\operatorname{mult}_{p}\left(V_{j}, H\right)$ is the intersection multiplicity of the component $V_{j}$ and the line $H$ at the point $p$.

Proof. We use the above exact sequence and get

$$
0 \rightarrow H^{1}\left(\mathcal{U}_{0}, \mathcal{L}\right) \rightarrow H^{1}(\mathcal{U}, \mathcal{L}) \rightarrow H^{0}\left(\mathcal{U}_{0} \cap H, \mathcal{L}\right) \rightarrow H^{2}\left(\mathcal{U}_{0}, \mathcal{L}\right) \rightarrow \ldots
$$

The existence of a point $p$ as stated implies that $H^{0}\left(\mathcal{U}_{0} \cap H, \mathcal{L}\right)=0$; hence clearly $\epsilon=0$ as well. If there is no such point $p$, then the local system $\mathcal{L} \mid\left(\mathcal{U}_{0} \cap H\right)$ is the trivial rank one local system $\mathbb{C}$ and hence $H^{0}\left(\mathcal{U}_{0} \cap H, \mathcal{L}\right)=\mathbb{C}$.

Remark 5.2. (added in proof) We point out that the converse statement in the second part of the above corollary is not true. Here is a counter-example. In $\mathbb{C P}^{2}$, let $V$ be the union of the following four lines: $x=0, x-z=0, y=0$ and $y-z=0$. Choose the line at infinity $H$ to be $z=0$. Let $\mathcal{L}$ be the local system with monodromy -1 about any of the first four lines, and trivial monodromy 1 about $H$. Then the product of the monodromies at each of the two intersection points $a=(0: 1: 0)$ and $b=(1: 0: 0)$ is 1 . On the other hand, both cohomology groups are trivial. For $H^{1}\left(\mathcal{U}_{0}, \mathcal{L}\right)$, we can use our Theorem 3.2 above, while for $H^{1}(\mathcal{U}, \mathcal{L})$ we 
can use the Künneth Formula (see Theorem 4.3.14 in [18]) with $X=Y=\mathbb{C} \backslash\{0,1\}$ and $\mathcal{F}=\mathcal{G}$ the rank one local system with monodromy -1 about the points 0 and 1 .

One case which is already well explored is the following.

Example 5.3. Assume that $n>1, s=1$ and that $V=V_{1}$ is a hypersurface of degree $d$ having only isolated singularities. Then $\pi_{1}\left(\mathcal{U}_{0}\right)=\mathbb{Z} / d \mathbb{Z}$ and hence a local system $\mathcal{L}=\mathcal{L}_{\beta}$ corresponds to a choice of a $d$-root of unity $\beta$. For $\beta=1$ we get $H^{0}\left(\mathcal{U}_{0} ; \mathbb{C}\right)=\mathbb{C}$ and $H^{j}\left(\mathcal{U}_{0} ; \mathbb{C}\right)=0$ for $0<j<n$. When $V$ is a $\mathbb{Q}$-manifold, one also has $H^{n}\left(\mathcal{U}_{0} ; \mathbb{C}\right)=0$. The computation of $H^{n}\left(\mathcal{U}_{0} ; \mathbb{C}\right) \approx H_{0}^{n+1}(V)$ is quite difficult in general, as it may depend on the position of the singularities; see [16], 32. Here $H_{0}^{*}(V)$ denotes the primitive cohomology of $V$, i.e., the cokernel of the natural monomorphism $H^{*}\left(\mathbb{C P}^{n+1}\right) \rightarrow H^{*}(V)$ induced by the inclusion of $V$ into $\mathbb{C P}^{n+1}$.

For $\beta \neq 1$, one can use the isomorphism $H^{m}\left(\mathcal{U}_{0}, \mathcal{L}\right)=H^{m}(F, \mathbb{C})_{\beta}$, the $\beta$ eigenspace of the monodromy acting on the Milnor fiber $F$ associated to $V$. In particular, $H^{m}\left(\mathcal{U}_{0}, \mathcal{L}\right)=0$ for $m<n$. It is possible to construct examples such that for $m \in\{n, n+1\}$ one has

$$
\operatorname{dim} H^{m}\left(\mathcal{U}_{0}, \mathcal{L}\right)>\operatorname{dim} H^{m}\left(\mathcal{U}_{0}, \mathbb{C}\right) .
$$

Indeed, consider the polynomials in [16, p. 148], which have a monodromy operator without the eigenvalue 1 on all the reduced cohomology groups $\tilde{H}^{m}(F, \mathbb{C})$ (equivalently, $V$ has the same rational cohomology as $\mathbb{C P}^{n}$ ). It is not possible that $\tilde{H}^{m}(F, \mathbb{C})=0$ for all $m \in \mathbb{N}$, by A'Campo's result on the Lefschetz number of the monodromy; see [18, p. 174]. Hence there is some integer $m$ and some $d$-root of unity $\beta \neq 1$ such that $\operatorname{dim} H^{m}\left(\mathcal{U}_{0}, \mathcal{L}_{\beta}\right)>0=\operatorname{dim} H^{m}\left(\mathcal{U}_{0}, \mathbb{C}\right)$. Using the Euler characteristic equality $\chi\left(\mathcal{U}_{0}, \mathbb{C}\right)=\chi\left(\mathcal{U}_{0}, \mathcal{L}_{\beta}\right)$, it follows that the inequality should hold for the two possible values of $m$. By the minimality property of hyperplane arrangement complements, it is known that the above inequality is impossible for such complements, 23.

5.1. Some 2-component arrangements. We consider now in detail the case of hypersurface arrangements $V$ with $s=2$ irreducible components. We assume moreover that:

(i) $n>1$ and each $V_{i}$ has at most isolated singularities and is a $\mathbb{Q}$-manifold;

(ii) $V^{\prime}=V_{1} \cap V_{2}$ has at most isolated singularities; this condition is automatically fulfilled when $d_{1}<d_{2}$ and $V_{2}$ is smooth, see 10 .

Let $\mathcal{U}_{i}=\mathbb{C P}^{n+1} \backslash V_{i}$. Then the Mayer-Vietoris sequence of the covering $\mathcal{U}^{\prime}=$ $\mathcal{U}_{1} \cup \mathcal{U}_{2}$ reads like

$$
\ldots \rightarrow H^{k-1}\left(\mathcal{U}_{0}\right) \rightarrow H^{k}\left(\mathcal{U}^{\prime}\right) \rightarrow H^{k}\left(\mathcal{U}_{1}\right) \oplus H^{k}\left(\mathcal{U}_{2}\right) \rightarrow H^{k}\left(\mathcal{U}_{0}\right) \rightarrow \ldots
$$

Here and in the sequel the constant coefficients $\mathbb{C}$ are used unless stated otherwise. Using Example 5.3 to handle the cohomology groups $H^{*}\left(\mathcal{U}_{i}\right)$ for $i=1,2$ and the Alexander duality isomorphism (which is compatible with the MHS after taking the Tate twist $(-n-1)$, see for details $[26])$

$$
H^{k}\left(\mathcal{U}^{\prime}\right)=H^{2 n+2-k}\left(\mathbb{C P}^{n+1}, V^{\prime}\right)^{\vee}(-n-1)=H_{0}^{2 n+1-k}\left(V^{\prime}\right)^{\vee}(-n-1),
$$

we get the following result. 
Proposition 5.4. With the above notation and assumptions, the following hold.

(i) $H^{0}\left(\mathcal{U}_{0}\right)=\mathbb{C}$ is pure of type $(0,0)$ and $H^{1}\left(\mathcal{U}_{0}\right)=\mathbb{C}$ is pure of type $(1,1)$ and it is spanned by the 1-form

$$
\omega_{1}=d_{2} \cdot \frac{d f_{1}}{f_{1}}-d_{1} \cdot \frac{d f_{2}}{f_{2}} .
$$

(ii) $H^{k}\left(\mathcal{U}_{0}\right)=0$ for $1<k<n$.

(iii) $H^{n}\left(\mathcal{U}_{0}\right)$ is pure of weight $n+2$ and $b_{n}\left(\mathcal{U}_{0}\right) \leq \operatorname{dim} H_{0}^{n}\left(V^{\prime}\right)$. Moreover $H^{n}\left(\mathcal{U}_{0}\right)=0$ if $d_{1}<d_{2}$ and $V_{2}$ is smooth.

(iv) $H^{n+1}\left(\mathcal{U}_{0}\right)$ has weights $\geq n+2$ and one has an isomorphism of $M H S$

$$
H^{n+1}\left(\mathcal{U}_{0}\right) / W_{n+2} H^{n+1}\left(\mathcal{U}_{0}\right)=H_{0}^{n-1}\left(V^{\prime}\right)^{\vee}(-n-1) .
$$

Proof. The vanishing of $H^{n}\left(\mathcal{U}_{0}\right)$ in the third claim follows from an unexpected source. Indeed, the Gysin sequence of the smooth divisor $X_{2}=V_{2} \cap \mathcal{U}_{1}$ in $\mathcal{U}_{1}$ gives a monomorphism $H^{n}\left(\mathcal{U}_{0}\right) \rightarrow H^{n-1}\left(X_{2}\right)$. But this latter group $H^{n-1}\left(X_{2}\right)$ is trivial by some general connectivity results recently obtained by the first author; see [15.

Indeed, Theorem 1.1 in [15] applied to $V=V_{2}$ and $H=V_{1}$ yields the $(n-1)$ connectivity of the relative Milnor fiber

$$
F_{\text {rel }}=\left\{x \in \mathbb{C}^{n+2} \mid f_{2}(x)=0, f_{1}(x)=1\right\} .
$$

Since $X_{2}$ is the quotient of $F_{r e l}$ under the obvious action of the group of $d_{1}$-roots of unity, we get $H^{n-1}\left(X_{2}\right)=0$.

The examples given in [15] show that the case $d_{1}=d_{2}$ is much more complicated; in particular, the group $H^{n-1}\left(X_{2}\right)$ can be nonzero. Example 5.6 below shows that the assumption $V_{2}$ smooth cannot be relaxed to $V_{2}$ with isolated singularities and a $\mathbb{Q}$-manifold. The key point here is that the singularities of $V_{2}$ are situated on $V_{1}$, a situation not covered by the results in [15].

The only other claims that are not obvious are those on the MHS. They follow from the fact that $H_{0}^{n}\left(V^{\prime}\right)$ has a pure HS of weight $n$ (the singularities of $V^{\prime}$ being isolated) and the following consequence of the Alexander duality (5.3)

$$
h^{p, q}\left(H^{k}\left(\mathcal{U}^{\prime}\right)\right)=h^{n+1-p, n+1-q}\left(H_{0}^{2 n+1-k}\left(V^{\prime}\right)\right) .
$$

For a rank one local system $\mathcal{L} \in \mathcal{L}\left(\mathcal{U}_{0}\right)$, we can choose the corresponding form $\omega_{\alpha}$ to be a multiple $a(\alpha) \omega_{1}$ of the 1 -form $\omega_{1}$ introduced above. Then Propositions 4.5 and 5.4 yield the following.

Corollary 5.5. For a nontrivial rank one local system $\mathcal{L} \in \mathcal{L}\left(\mathcal{U}_{0}\right)$ for which an admissible choice of residues $\alpha=\left(d_{2} \cdot a(\alpha),-d_{1} \cdot a(\alpha)\right)$ exists, the following hold:

(i) $H^{k}\left(\mathcal{U}_{0}, \mathcal{L}\right)=0$ for $k<n$;

(ii) if $H_{0}^{n}\left(V^{\prime}\right)=0$ or if $d_{1}<d_{2}$ and $V_{2}$ is smooth, then $H^{n}\left(\mathcal{U}_{0}, \mathcal{L}\right)=0$.

Note that the first claim above holds by Corollary 3.5 , since $V^{\prime}$ has only INNC singularities.

The vanishing of $H_{0}^{n}\left(V^{\prime}\right)$ holds when $V^{\prime}$ is a $\mathbb{Q}$-homology manifold, but also in many other cases; see for instance the discussion in [16, pp. 207-216]. There one considers only the case when $V_{1}$ is a hyperplane. Indeed, any hypersurface $W$ having only isolated singularities in $\mathbb{C P}^{n}$ can be obtained as the intersection of a smooth hypersurface $V_{2}$ in $\mathbb{C P}^{n+1}$ with the hyperplane $H=\mathbb{C P}^{n}$; see [14, p. 206]. 
However, this situation is usually uninteresting according to the second claim of the above corollary.

We conclude with an example where $V_{1}$ is a hyperplane and $V_{2}$ is singular, so that it may have been considered already in the previous section (in such a case $\mathcal{U}_{0}$ from this section is exactly $\mathcal{U}$ from the previous section, but for the hypersurface $\left.V=V_{2} !\right)$.

Example 5.6. In $\mathbb{C P}^{3}$ (with homogeneous coordinates $(x: y: z: t)$ ) consider the hyperplane $H=V_{1}: t=0$ and the surface $V_{2}: x y z-t^{3}=0$. Then $V_{2}$ has exactly 3 singularities of type $A_{2}$; hence it is a $\mathbb{Q}$-manifold. Moreover, $H$ is transverse to $V_{2}$, except at the 3 singular points of $V_{2}$.

To compute the cohomology of the complement $\mathcal{U}_{0}$, we use the Gysin exact sequence of the smooth divisor $D=V_{2} \backslash V_{1}$ in the affine space $\mathbb{C P}^{3} \backslash V_{1}$ (with coordinates $(x, y, z))$ and get

$$
H^{k}\left(\mathcal{U}_{0}\right)=H^{k-1}(D)(-1)
$$

for $k=2,3$, where $(-1)$ denotes the Tate twist. Now $D$ is given by the equation $x y z=1$; hence it is a 2-dimensional torus. It follows that

(i) $H^{2}\left(\mathcal{U}_{0}\right)=\mathbb{C}^{2}$ is pure of type $(2,2)$;

(ii) $H^{3}\left(\mathcal{U}_{0}\right)=\mathbb{C}$ is pure of type $(3,3)$. Moreover, as explained in the fourth section, the 1 -form $\omega_{1}$ is a multiple of $\frac{d g}{g}$ with $g=x y z-1$.

Let $g_{0}=g+1=x y z$, and note that $F_{g_{0}}=D$ is the Milnor fiber of the homogeneous polynomial $g_{0}$. Since $\mathcal{U}_{0}=\mathbb{C}^{3} \backslash F_{g_{0}}$, we may use the description of the cohomology groups of $\mathcal{U}_{0}$ using Remark (2.11) in [16], p.192. By taking

$$
\eta_{i}=A_{i} x d y \wedge d z-B_{i} d x \wedge d z+C_{i} d x \wedge d y
$$

in the formula (2.12) loc. cit. with $\left(A_{1}, B_{1}, C_{1}\right)=(x,-y, 0)$ and $\left(A_{2}, B_{2}, C_{2}\right)=$ $(0,-y, z)$ we get a basis of $H^{2}\left(\mathcal{U}_{0}\right)$. A direct computation then shows that $\omega_{1} \wedge \eta_{i}=0$ in $H^{3}\left(\mathcal{U}_{0}\right)=H^{2}(D)$. To see this, note that $d \eta_{i}=d g \wedge \eta_{i}=0$ and hence the Poincaré-Leray residue of the form

$$
\omega_{1} \wedge \eta_{i}=\frac{d g}{g} \wedge \eta_{i}
$$

is the form $\eta_{i}$. Since $d \eta_{i}=0$ on $\mathbb{C}^{3}$, it follows that $\eta_{i}=d \eta_{i}^{\prime}$, for some 1 -forms $\eta_{i}^{\prime}$ on $\mathbb{C}^{3}$. Hence the cohomology class of $\eta_{i}$ in $H^{2}(D)$ is trivial.

It follows that, for a nontrivial rank one local system $\mathcal{L} \in \mathcal{L}\left(\mathcal{U}_{0}\right)$ for which an admissible choice of residues $\alpha$ exists, one has $H^{*}\left(\mathcal{U}_{0}, \mathcal{L}\right)=H^{*}\left(H^{*}\left(\mathcal{U}_{0}\right), \omega_{\alpha}\right)$. Therefore we get the following equalities:

$$
\operatorname{dim} H^{0}\left(\mathcal{U}_{0}, \mathcal{L}\right)=\operatorname{dim} H^{1}\left(\mathcal{U}_{0}, \mathcal{L}\right)=0, \operatorname{dim} H^{2}\left(\mathcal{U}_{0}, \mathcal{L}\right)=2, \text { and } \operatorname{dim} H^{3}\left(\mathcal{U}_{0}, \mathcal{L}\right)=1 .
$$

In particular, $\operatorname{Supp} A^{2}\left(\mathcal{U}_{0}\right)$ coincides with the character torus $\mathbb{T}^{1}$. This follows since $\operatorname{Supp} A^{2}\left(\mathcal{U}_{0}\right)$ is a Zariski closed subset, with a nonempty interior by Remark 4.4. in the irreducible algebraic variety $\mathbb{T}^{1}$. The reader should compare this fact to Theorem 3.6 above.

\section{REFERENCES}

1. Arapura, D., Geometry of cohomology support loci for local systems. I. J. Algebraic Geom. 6 (1997), 563-597. MR 1487227 (98m:32043)

2. Borel, A., Spaltenstein, N. Sheaf theoretic intersection cohomology, Progress in Mathematics, vol. 50, Birkhäuser, Boston, 1984. MR0788171 (88d:32024) 
3. Cimasoni, D., Studying the multivariable Alexander polynomial by means of Seifert surfaces, Bol. Soc. Mat. Mexicana (3) 10 (2004), Special Issue, 107-115. MR2199342 (2006j:57011)

4. Cogolludo-Agustín, J.I., Topological invariants of the complement to arrangements of rational plane curves. Mem. Amer. Math. Soc. 159 (2002), no. 756, xiv+75 pp. MR 1921584 (2003k:32037)

5. Cohen, D.C., Orlik, P., Arrangements and local systems, Math. Research Letters 7 (2000), 299-316. MR1764324 (2001i:57040)

6. Cohen, D. C., Suciu, A. I., Alexander invariants of complex hyperplane arrangements, Transactions of the AMS, Vol. 351 (10), 4043-4067, (1999). MR.1475679 (99m:52019)

7. Cohen, D. C., Suciu, A. I., Characteristic varieties of arrangements, Math. Proc. Cambridge Philos. Soc. 127(1999), 33-54. MR.1692519 (2000m:32036)

8. Cohen, D. C, Denham G. and Suciu, A.I., Torsion in Milnor fiber homology, Algebraic and Geometric Topology 3 (2003), 511-535. MR 1997327 (2004d:32043)

9. Cohen, D.C., Dimca, A., Orlik, P., Nonresonance conditions for arrangements, Ann. Inst. Fourier, Grenoble, 53, 6 (2003), 1883-1896. MR2038782(2005a:32027)

10. Choudary, A.D.R. and Dimca, A., Hypersurface singularities, codimension two complete intersections and tangency sets, Geom. Dedicata 24 (1987), 255-260. MR0914821 (89d:14017)

11. Davis, J.F., Kirk, P., Lecture Notes in Algebraic Topology, Graduate Studies in Mathematics, Vol.35, AMS 2001. MR1841974 (2002f:55001)

12. Deligne, P., Equations différentielles à points singuliers réguliers, Lecture Notes in Math., 163, Springer, Berlin (1970). MR0417174 (54:5232)

13. Deligne, P., Théorie de Hodge II. Publ. Math. IHES, 40, 5-57 (1971). MR0498551(58:16653a)

14. Dimca, A., Topics on Real and Complex Singularities, Vieweg Advanced Lectures in Mathematics, Friedr. Vieweg und Sohn, Braunschweig, 1987. MR.1013785 (92d:32048)

15. Dimca, A., On the connectivity of some complete intersections, arXiv:math.AG/0507501.

16. Dimca, A., Singularities and Topology of Hypersurfaces, Universitext, Springer-Verlag, 1992. MR:1194180 (94b:32058)

17. Dimca, A., Hyperplane arrangements, M-tame polynomials and twisted cohomology, in: Commutative Algebra, Singularities and Computer Algebra, Eds. J. Herzog, V. Vuletescu, NATO Science Series, Vol. 115, Kluwer 2003, pp. 113-126. MR2030266 (2005b:32062)

18. Dimca, A., Sheaves in Topology, Universitext, Springer-Verlag, 2004. MR2050072 (2005j:55002)

19. Dimca, A., Lehrer, G.I., Purity and equivariant weight polynomials, in: Algebraic Groups and Lie Groups, editor G.I. Lehrer, Cambridge University Press, 1997. MR1635679 (99h:14023)

20. Dimca, A., Nemethi, A., Hypersurface complements, Alexander modules and Monodromy, Proceedings of the 7th Workshop on Real and Complex Singularities, Sao Carlos, 2002, Amer. Math. Soc (2004). MR2087802 (2005h:32076)

21. Dimca, A., Libgober, A., Regular functions transversal at infinity, arXiv: math.AG/0504128, to appear in Tohoku Math.J.

22. Dimca, A., Libgober, A., Local topology of reducible divisors, arXiv: math.AG/0303215.

23. Dimca, A., Papadima, S., Hypersurface complements, Milnor fibers and higher homotopy groups of arrangements, Annals of Math. 158 (2003), 473-507. MR2018927(2005a:32028)

24. Eisenbud, D., Neumann, W., Three-dimensional link theory and invariants of plane curve singularities, Annals of Math. Studies 110, Princeton University Press, Princeton, 1985. MR0817982 (87g:57007)

25. Esnault, H., Schechtman, V., Viehweg, E.: Cohomology of local systems on the complement of hyperplanes. Invent. Math., 109, 557-561 (1992). Erratum, ibid. 112, 447 (1993). MR 1176205 (93g:32051) MR 1213111 (94b:32061)

26. Fujiki, A., Duality of mixed Hodge structures of algebraic varieties, Publ. RIMS Kyoto Univ. 16 (1980), 635-667. MR0602463 (82h:14007)

27. Goresky, M., MacPherson, R., Intersection homology II, Invent. Math. 72 (1983), 77-129. MR0696691 (84i:57012)

28. Hartshorne, R., Algebraic Geometry, Graduate Texts in Mathematics 52, Springer, 1977. MR 0463157 (57:3116)

29. Hatcher, A., Algebraic Topology, Cambridge University Press, 2002. MR 1867354 (2002k:55001)

30. Hillman, J. A., Alexander ideals of links, Lecture Notes in Math. 895, Springer, 1981. MR:0653808 (84j:57004) 
31. Levine, J., Knot Modules, I, Transactions of the A.M.S., 229 (1977), 1-50. MR0461518 $(57: 1503)$

32. Libgober, A., Homotopy groups of the complements to singular hypersurfaces, II, Annals of Mathematics, 139 (1994), 117-144. MR1259366 (95d:14023)

33. Libgober, A., Hodge decomposition of Alexander invariants, Manuscripta Math., 107 (2002), 251-269. MR1894743 (2003f:32041)

34. Libgober, A., On the homology of finite abelian coverings, Topology and its Applications, 43 (1992) 157-166. MR1152316 (93e:57003)

35. Libgober, A., Characteristic varieties of algebraic curves arXiv: math.AG/9801070, in: C. Ciliberto et al. (eds.), Applications of Algebraic Geometry to Coding Theory, Physics and Computation, 215-254, Kluwer, 2001. MR1866902 (2003e:14008)

36. Libgober, A., Isolated non-normal crossing, in Real and Complex Singularities, 145-160, Contemporary Mathematics, 354, 2004. MR2087810 (2005k:32035)

37. Libgober, A., Homotopy groups of complements to ample divisors, arXiv: math.AG/0404341, to appear in Proceedings of 12th MSJ-IRI symposium "Singularity theory and its applications" Hokkaido Univerisity.

38. Libgober, A., Yuzvinsky, S., Cohomology of the Orlik-Solomon algebras and local systems, Compositio Math. 121 (2000), 337-361. MR1761630(2001j:52032)

39. Loeser, F. and Vaquié, M.: Le polynôme d'Alexander d'une courbe plane projective, Topology 29 (1990),163-173. MR1056267 (91d:32053)

40. Massey, D. B., Introduction to perverse sheaves and vanishing cycles in Singularity Theory, ICTP 1991, Eds. D.T. Le, K. Saito, B. Teissier, 487-509. MR.1378418 (96m:32047)

41. Matei, D. and Suciu, A., Hall invariants, homology of subgroups, and characteristic varieties, Internat. Math. Res. Notices 9 (2002), 465-503. MR1884468 (2003d:20055)

42. Maxim, L., Intersection homology and Alexander modules of hypersurface complements, Comm. Math. Helv., Vol 81 (1), 2006, pp. 123-155. MR2208801

43. Milnor, J., Singular points of complex hypersurfaces, Annals of Mathematical Studies 61, vol. 50, Princeton University Press, Princeton, 1968. MR0239612 (39:969)

44. Milnor, J., Infinite cyclic coverings, Topology of Manifolds, Boston, 1967. MR0242163 $(39: 3497)$

45. Milnor, J., A duality theorem for Reidemeister torsion, Ann. of Math. 76 (1962), 137-147. MR0141115 (25:4526)

46. Randell, R.: Milnor fibers and Alexander polynomials of plane curves, Proc. Symp. Pure Math. 40, Part 2., AMS 1983, pp. 415-419. MR0713266 (85h:14018)

47. Schechtman, V., Terao, H., Varchenko, A.: Local systems over complements of hyperplanes and the Kac-Kazhdan condition for singular vectors. J. Pure Appl. Algebra, 100, 93-102 (1995). MR 1344845 (96j:32047)

48. Schurmann, J., Topology of Singular Spaces and Constructible Sheaves, Birkhäuser, Monografie Matematyczne 63, 2003. MR 2031639 (2005f:32053)

49. Suciu, A. I., Translated tori in the characteristic varieties of complex hyperplane arrangements. Arrangements in Boston: a Conference on Hyperplane Arrangements (1999). Topology Appl. 118 (2002), no. 1-2, 209-223. MR1877726 (2002j:32027)

50. Sumners, D.W., Woods, J.M., The monodromy of reducible plane curves, Inventiones Math. 40, 107-141 (1977). MR0458440 (56:16643)

51. Weibel, C.A.: An Introduction to Homological Algebra, Cambridge Studies in Advanced Math., 38, Cambridge Univ. Press, Cambridge (1994). MR.1269324 (95f:18001)

Laboratoire J.A. Dieudonné, UMR du CNRS 6621, Université de Nice-Sophia Antipolis, Parc Valrose, 06108 Nice Cedex 02, France

E-mail address: dimca@math.unice.fr

Institute of Mathematics of the Romanian Academy, P.O. Box 1-764, 70700 Bucharest, ROMANIA

Current address: Department of Mathematics, University of Illinois at Chicago, $851 \mathrm{~S}$ Morgan Street, Chicago, Illinois, 60607

E-mail address: lmaxim@math.uic.edu 